\title{
Defect topologies in chiral liquid crystals confined to mesoscopic channels
}

Cite as: J. Chem. Phys. 142, 194704 (2015); https://doi.org/10.1063/1.4920979

Submitted: 04 December 2014 . Accepted: 30 April 2015 . Published Online: 19 May 2015

Sergej Schlotthauer, Robert A. Skutnik, Tillmann Stieger, and Martin Schoen
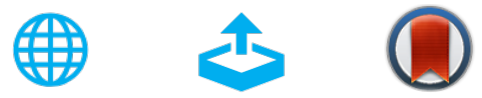

View Online

Export Citation

CrossMark

\section{ARTICLES YOU MAY BE INTERESTED IN}

A novel model for smectic liquid crystals: Elastic anisotropy and response to a steady-state flow

The Journal of Chemical Physics 145, 164903 (2016); https://doi.org/10.1063/1.4965711

Defect topologies in a nematic liquid crystal near a patchy colloid

The Journal of Chemical Physics 136, 194703 (2012); https://doi.org/10.1063/1.4717619

Effects of flow on topological defects in a nematic liquid crystal near a colloid

The Journal of Chemical Physics 140, 054905 (2014); https://doi.org/10.1063/1.4862953

Lock-in Amplifiers Find out more today

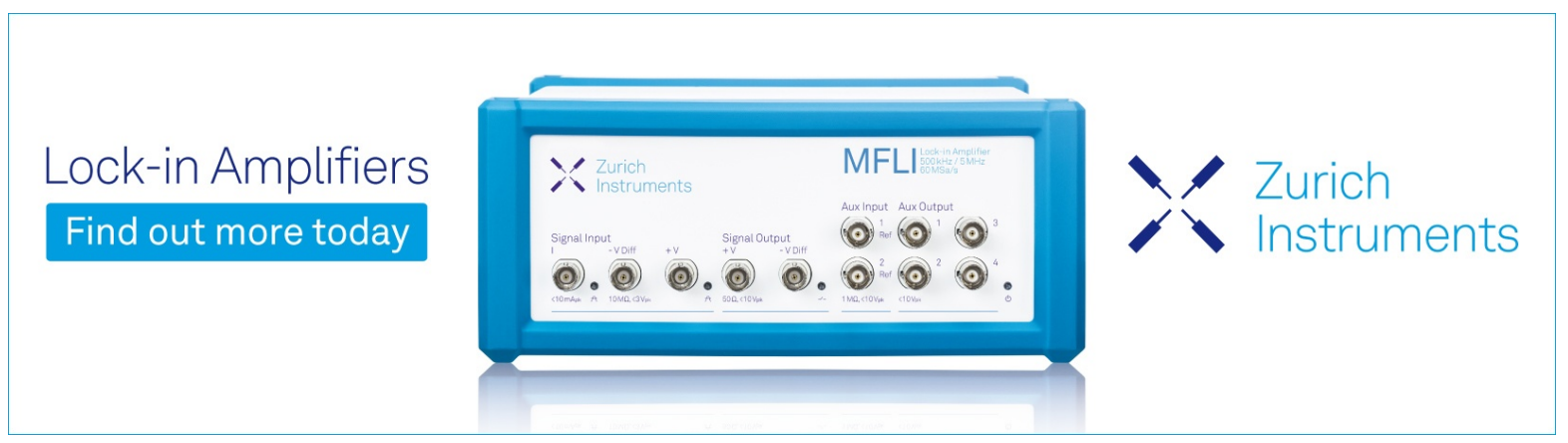

Zurich

Instruments 


\title{
Defect topologies in chiral liquid crystals confined to mesoscopic channels
}

\author{
Sergej Schlotthauer, ${ }^{1, a)}$ Robert A. Skutnik, ${ }^{1}$ Tillmann Stieger, ${ }^{1}$ and Martin Schoen ${ }^{1,2}$ \\ ${ }^{1}$ Stranski-Laboratorium für Physikalische und Theoretische Chemie, Technische Universität Berlin, Sekr. C7, \\ Straße des 17. Juni 135, Berlin 10623, Germany \\ ${ }^{2}$ Department of Chemical and Biomolecular Engineering, North Carolina State University, 911 Partners Way, \\ Raleigh, North Carolina 27695, USA
}

(Received 4 December 2014; accepted 30 April 2015; published online 19 May 2015)

\begin{abstract}
We present Monte Carlo simulations in the grand canonical and canonical ensembles of a chiral liquid crystal confined to mesochannels of variable sizes and geometries. The mesochannels are taken to be quasi-infinite in one dimension but finite in the two other directions. Under thermodynamic conditions chosen and for a selected value of the chirality coupling constant, the bulk liquid crystal exhibits structural characteristics of a blue phase II. This is established through the tetrahedral symmetry of disclination lines and the characteristic simple-cubic arrangement of double-twist helices formed by the liquid-crystal molecules along all three axes of a Cartesian coordinate system. If the blue phase II is then exposed to confinement, the interplay between its helical structure, various anchoring conditions at the walls of the mesochannels, and the shape of the mesochannels gives rise to a broad variety of novel, qualitative disclination-line structures that are reported here for the first time. ( 92015 AIP Publishing LLC. [http://dx.doi.org/10.1063/1.4920979]
\end{abstract}

\section{INTRODUCTION}

Liquid crystals are a fascinating class of soft matter systems that are still receiving a lot of interest from the scientific community even after more than one and a halfcenturies of research. ${ }^{1}$ In more recent times, the focus has shifted towards liquid crystals composed of chiral molecules (i.e., mesogens). The interesting properties of this class of liquid crystals arise from the combination of handedness of the interaction between a pair of mesogens and their ability to form (orientationally and/or positionally) ordered phases with helical substructures. ${ }^{2}$

Among the various phases that are formed by chiral liquid crystals, the so-called blue phases are of particular interest. This is because they exhibit spectacular reflections of visible light that can be controlled by external fields. Blue phases have therefore been considered as tunable photonic materials with a wide range of potential applications. . $^{3,4}$

Unfortunately, when it comes to applications, blue phases turned out to be rather difficult to handle. This is because in the past and under ordinary conditions, their thermal stability was rather limited and amounted only to a few $\mathrm{K}$. Hence, from a practical perspective, it is highly desirable to broaden the regime of thermal stability of blue phases considerably. ${ }^{5}$ In the meantime, a lot of progress has been made to enhance this stability range. For example, Kikuchi et al. ${ }^{6}$ could stabilize blue phases by polymers thus widening the temperature range of stable blue phase II to $60 \mathrm{~K}$. An even larger range of stability of $-125^{\circ} \mathrm{C}$ to $125^{\circ} \mathrm{C}$ has been reported by Castles et $a l .^{7}$ who investigated the blue-phase templated fabrication of nanostructures for photonic application. Other methods to

\footnotetext{
${ }^{a)}$ Electronic mail: s.schlotthauer@mailbox.tu-berlin.de
}

stabilize blue phases have been reported by Zheng et al. ${ }^{8} \mathrm{He}$ et al. ${ }^{9}$ and Yoshizawa et al. ${ }^{10}$

Similar advances have been made to reduce the pitch $p$ of the double-twist helices characteristic of blue phases. Whereas traditionally $p$ is of the order of $\mu \mathrm{m}$, Oo et al ${ }^{11}$ have recently reported materials where $p$ is only about $160 \mathrm{~nm}$.

The key structural characteristic of blue phases is a complex network of (double-twist) helices such that locally blue phases are highly ordered entities whereas globally they appear to be isotropic, that is, they do not exhibit birefringence, for example. Because of the high degree of local order and the global isotropy, many regions exist in blue phases where mesogens are orientationally frustrated. These regions form disclination lines that exhibit geometrically highly non-trivial, three-dimensional structures.

If colloidal particles are dissolved in blue phases, the colloids experience structural forces from the disclination lines that allow one to build and stabilize unique spatial arrangements of the colloids. ${ }^{12-14}$ The possibility to arrange colloidal particles through effective interactions with a unique, locally ordered host phase is of immense fundamental and technological interest. This is because the colloidal suspension may exhibit properties not present in the pure bulk compound as a consequence of the interaction between the (nano-)colloidal objects and their anisotropic host. Moreover, differently structured host phases may be used to drive the assisted and directed self-assembly of nanoparticle arrangements in particular ways.

If, in addition, external fields are applied, one may switch between various structures of the liquid crystal which enhances the number of ways in which dispersed colloidal particles may be arranged. The field-induced structures in blue phases and their dynamics have recently been explored by Fukuda and Žumer using Landau-de Gennes continuum theory. ${ }^{15}$ 
More recently, quite a bit of experimental and theoretical interest has been devoted to study torons and other soliton-like structures. ${ }^{16-20}$ These rather complex structures are created and stabilized in external laser fields.

Another realization of such an external field is the wall of a nanocontainer to which the liquid crystal is confined. These nanoconfining containers such as nanopores of various widths and geometries can nowadays be synthesized almost routinely in the laboratory in a controlled fashion and with specifically tailored properties. ${ }^{21-24}$ Confined chiral liquid crystals have been studied experimentally ${ }^{25-27}$ and theoretically. ${ }^{25-29}$ On the theory side, continuum approaches based upon a minimization of the Frank free-energy density ${ }^{25-27,29}$ or homotopy theory ${ }^{28}$ have been employed to study the defect topologies in confined chiral liquid crystals. To the best of our knowledge, this work is the first molecule-based theoretical study of such topologies.

In particular, confinement stabilizes unique structures in chiral liquid-crystalline phases. ${ }^{19}$ This has been shown experimentally quite some time ago ${ }^{30}$ but a comprehensive theoretical understanding of the interplay between chirality and confinement is still in its infancy. For example, focusing on blue phase I confined to a slit-pore, Fukuda and Žumer use Landau-de Gennes theory to investigate novel defect structures under severe confinement. ${ }^{31}$ Within the framework of Landau-de Gennes theory, these same authors observed ring defects where these rings are independent entities that do not intersect in space and form very beautiful, highly symmetric structures. $^{32}$

From a molecular point of view, very little effort has been invested so far to study chiral liquid crystals under confinement conditions. One reason for the relative lack of such molecule-based studies may be that the size of the pitch of helical structures forming is usually too large to be captured at the resolution of individual mesogens. This is not so for the model system employed in this and previous studies by some of us. ${ }^{33,34}$

Our model consists of a Lennard-Jones core with superimposed orientation dependent dispersion attraction combined with a certain handedness of these interactions. In fact, as we showed earlier, this model is capable of forming blue phases II and III besides a cholesteric phase. ${ }^{33}$ In particular, the blue phase II could be identified unambiguously through its characteristic double-twist helices arranged in a simple-cubic lattice and the tetrahedral arrangement of its disclination lines. For colloids placed in the cholesteric phase of this model, disclination lines near the colloid's surface could be observed in excellent qualitative agreement with lattice Boltzmann studies $^{35}$ and even with experimental data ${ }^{36}$ both of which focus on length scales much larger than those characteristic of our model. However, a direct quantitative comparison with these earlier studies ${ }^{35,36}$ is precluded because upscaling defect topologies to larger length scales would only be possible for nonsingular defects.

The remainder of our manuscript is organized as follows. In Sec. II, we give a brief introduction to properties that we seek to compute. Section III is given to an introduction of our model of a confined chiral liquid crystal. Results of this study are presented in Sec. IV. The paper concludes in Sec. V with a summary of our findings.

\section{PROPERTIES}

In this work, we focus on the interplay between the geometry of mesochannels and structural properties of ordered liquid crystals composed of chiral molecules. This interplay gives rise to interesting and unique disclination lines in the confined liquid crystal that have no immediate counterpart in the bulk. From an operational point of view, we define disclination lines through the set

$$
\ell \equiv\left\{\boldsymbol{r}=(x, y, z)^{\mathrm{T}} \mid S(\boldsymbol{r}) \leq \delta_{\mathrm{S}}\right\},
$$

where the superscript $\mathrm{T}$ denotes the transpose of the threedimensional vector $\boldsymbol{r}, S(\boldsymbol{r})$ is the local nematic order parameter at point $\boldsymbol{r}$, and $\delta_{\mathrm{S}}$ is a threshold value for $S(\boldsymbol{r})$. Clearly, the value of $\delta_{\mathrm{S}}$ is unknown a priori. It has to be chosen to optimize the visibility of $\ell$. In practice, it turns out that setting $\delta_{\mathrm{S}}=0.20$ provides an optimal representation of $\ell$ so that we fix $\delta_{\mathrm{S}}$ to this value throughout the present study.

Because of Eq. (2.1), the key quantity to be computed is the local nematic order parameter $S(\boldsymbol{r})$. It can, in principle, be defined as the ensemble average of the second Legendre polynomial. However, this definition is useless from a computational perspective because the nematic director is unknown $a$ priori as has been discussed quite some time ago by Eppenga and Frenkel. ${ }^{37}$ These authors suggest to consider the alignment tensor ${ }^{38}$ instead.

Here, we are particularly interested in the local alignment tensor defined at the microscopic level as

$\mathbf{Q}(\boldsymbol{r}) \equiv \frac{1}{2 \rho(\boldsymbol{r})}\left\langle\sum_{i=1}^{N}\left[3 \widehat{\boldsymbol{u}}\left(\omega_{i}\right) \otimes \widehat{\boldsymbol{u}}\left(\omega_{i}\right)-\mathbf{1}\right] \delta\left(\boldsymbol{r}-\boldsymbol{r}_{i}\right)\right\rangle$,

where

$$
\rho(\boldsymbol{r})=\left\langle\sum_{i=1}^{N} \delta\left(\boldsymbol{r}-\boldsymbol{r}_{i}\right)\right\rangle
$$

is the local average number density, the operator " $\otimes$ " stands for the tensor product, $\mathbf{1}$ is the unit tensor, angular brackets $\langle\ldots\rangle$ denote an ensemble average, $\delta(\ldots)$ is the Dirac $\delta$ function, and the caret is used to indicate a unit vector throughout this manuscript. In particular, $\widehat{\boldsymbol{u}}\left(\omega_{i}\right)$ is a unit vector specifying the orientation of mesogen $i$ in a space-fixed frame of reference. On account of the uniaxial symmetry of the mesogens, $\omega_{i}=\left(\phi_{i}, \theta_{i}\right)$ where $\phi_{i}$ and $\theta_{i}$ are two (out of three) Euler angles.

According to its definition, $\mathbf{Q}$ can be represented by a $3 \times 3$ matrix that is symmetric, traceless, and real. It satisfies the eigenvalue equation

$$
\mathbf{Q}(\boldsymbol{r}) \widehat{\boldsymbol{n}}_{i}(\boldsymbol{r})=\lambda_{i}(\boldsymbol{r}) \widehat{\boldsymbol{n}}_{i}(\boldsymbol{r}), \quad i=1, \ldots, 3,
$$

where $\widehat{\boldsymbol{n}}_{i}$ and $\lambda_{i}$ are the $i$ th eigenvector and eigenvalue of $\mathbf{Q}$, respectively. Using Jacobi's method, ${ }^{39} \mathbf{Q}$ can be diagonalized numerically in the basis of its three eigenvectors. Following previous workers, ${ }^{37,40-42}$ we take

$$
S(\boldsymbol{r}) \equiv \max [\operatorname{diag} \mathbf{Q}(\boldsymbol{r})]=\max _{i=1, \ldots, 3} \lambda_{i}(\boldsymbol{r})
$$

as a definition of the local nematic order parameter where $\operatorname{diag} \mathbf{Q}(\boldsymbol{r})$ is the diagonalized local alignment tensor. The 
eigenvector associated with the largest eigenvalue is then taken as the local nematic director $\widehat{\boldsymbol{n}}(\boldsymbol{r})$.

Another quantity that allows us to characterize our model liquid crystal is the nematic correlation length $\xi$. For the bulk system, we define a correlation function

$$
g_{2}\left(r_{\|}\right) \equiv\left\langle\frac{1}{N\left(r_{\|}\right)} \sum_{i=1}^{N} \sum_{\substack{j=1 \\ i \neq j}}^{N} P_{2}\left[\widehat{\boldsymbol{u}}\left(\omega_{i}\right) \cdot \widehat{\boldsymbol{u}}\left(\omega_{j}\right)\right] \delta\left(r_{\|}-r_{i j}^{\|}\right)\right\rangle,
$$

where $P_{2}$ is the second Legendre polynomial, $r_{i j}^{\|} \equiv\left|\boldsymbol{r}_{i}^{\|}-\boldsymbol{r}_{j}^{\|}\right|$, $\boldsymbol{r}_{i, j}^{\|} \equiv\left[\boldsymbol{r}_{i, j} \cdot \widehat{\boldsymbol{u}}\left(\omega_{i, j}\right)\right] \widehat{\boldsymbol{u}}\left(\omega_{i, j}\right)$, and $N\left(r_{\|}\right)$is the number of particles located in a cylindrical shell of a certain radius and height (see Sec. IV B) centered on $\boldsymbol{r}_{i}^{\|}$and oriented such that the cylinder axis is parallel to $\boldsymbol{r}_{i}^{\|}$. In the limit of sufficiently large $r_{\|}$, this correlation function allows us to estimate $\xi$ as described in Sec. IV B. We note in passing that our definition of $g_{2}$ in Eq. (2.6) is analogous to the one used earlier by Memmer et al. ${ }^{43}$

\section{MODEL SYSTEM}

For the current model of a confined liquid crystal, each configuration of the $N$ mesogens is associated with a total configurational potential energy that can be written as

$$
\Phi(\boldsymbol{R}, \boldsymbol{\Omega})=\Phi_{\mathrm{mm}}(\boldsymbol{R}, \boldsymbol{\Omega})+\Phi_{\mathrm{mw}}(\boldsymbol{R}, \boldsymbol{\Omega}),
$$

where subscripts "mm" and "mw" refer to mesogen-mesogen and mesogen-wall contributions, respectively. Here, $\boldsymbol{R}$ $=\left\{\boldsymbol{r}_{1}, \boldsymbol{r}_{2}, \ldots, \boldsymbol{r}_{N}\right\}$ and $\boldsymbol{\Omega}=\left\{\omega_{1}, \omega_{2}, \ldots, \omega_{N}\right\}$ denote sets of the center-of-mass coordinates and the polar angles describing positions and orientations of the mesogens, respectively.

\section{A. The liquid crystal in the bulk}

We begin the discussion with $\Phi_{\mathrm{mm}}$ assuming pairwise additivity of the mesogen-mesogen interactions so that

$$
\Phi_{\mathrm{mm}}(\boldsymbol{R}, \boldsymbol{\Omega})=\frac{1}{2} \sum_{i=1}^{N} \sum_{\substack{j=1 \\ j \neq i}}^{N} \varphi_{\mathrm{mm}}\left(\boldsymbol{r}_{i j}, \omega_{i}, \omega_{j}\right)
$$

where $\boldsymbol{r}_{i j} \equiv \boldsymbol{r}_{i}-\boldsymbol{r}_{j}$ and $\varphi_{\mathrm{mm}}$ is the mesogen-mesogen interaction potential. The latter is split into an isotropic and into an anisotropic contribution according to

$$
\varphi_{\mathrm{mm}}\left(\boldsymbol{r}_{i j}, \omega_{i}, \omega_{j}\right)=\varphi_{\mathrm{iso}}\left(r_{i j}\right)+\varphi_{\mathrm{anis}}\left(\boldsymbol{r}_{i j}, \omega_{i}, \omega_{j}\right),
$$

where $r_{i j}=\left|\boldsymbol{r}_{i j}\right|$. For the isotropic part, we adopt the wellknown Lennard-Jones potential

$$
\varphi_{\mathrm{iso}}\left(r_{i j}\right)=4 \varepsilon_{\mathrm{mm}}\left[\left(\frac{\sigma}{r_{i j}}\right)^{12}-\left(\frac{\sigma}{r_{i j}}\right)^{6}\right],
$$

where $\varepsilon$ is the depth of the attractive well.

In view of the nearly spherically symmetric shape of $\varphi_{\mathrm{mm}}$ (see, for example, Fig. 1 of Ref. 41 for the achiral version of our model), it has been suggested by Steuer et al. ${ }^{44}$ that $\sigma$ should be viewed as the typical size of a nearly spherical assembly of individual mesogens in the spirit of ideas originally put forward by Maier and Saupe. ${ }^{45}$ Hence, in this case $\sigma$ should be of the order of $1-10 \mathrm{~nm}$. As we will demonstrate below in Sec. IV B, this brings the pitch $p$ of helical structures forming under the conditions adopted here well within reach of $p \approx 100 \mathrm{~nm}$ observed in the recent experiments of Oo et al. ${ }^{11}$ Nevertheless, it needs to be emphasized that this $p$ is typically two or three orders of magnitude smaller than typical values found traditionally in experimental studies. ${ }^{46,47}$

To treat the anisotropic part of $\varphi_{\mathrm{mm}}$, we follow Giura and Schoen $^{48}$ and expand $\varphi_{\text {anis }}$ in the basis of rotational invariants $\Phi_{l_{i} l_{j} l}$ according to

$\varphi_{\text {anis }}\left(\boldsymbol{r}_{i j}, \omega_{i}, \omega_{j}\right)=\sum_{l_{i} l_{j} l} \varphi_{l_{i} l_{j} l}\left(r_{i j}\right) \Phi_{l_{i} l_{j} l}\left(\omega_{i}, \omega_{j}, \omega\right)$,

where $l_{i}, l_{j}$, and $l$ are non-negative integers and $\omega$ is the orientation of $\widehat{\boldsymbol{r}}_{i j} \equiv \boldsymbol{r}_{i j} / r_{i j}$. Restricting the discussion to dispersion interactions, the expansion coefficients $\varphi_{l_{i} l_{j} l}$ are proportional to $r_{i j}^{-6}$ regardless of $l_{i}, l_{j}$, and $l^{49}$

To specify the integers $l_{i}, l_{j}$, and $l$, we notice that many liquid crystals possess head-tail symmetry, that is, the interaction potential should remain invariant if one replaces either $\omega_{i}$ by $-\omega_{i}$ or $\omega_{j}$ by $-\omega_{j}$ (see, for example, pp. 3-10 of Ref. 2). Because of the definition of rotational invariants in terms of spherical harmonics and because of the parity rule $^{49}$ for the latter set of functions, $l_{i}$ and $l_{j}$ are immediately restricted to zero or even integers. ${ }^{48}$

However, integers $l_{i}, l_{j}$, and $l$ are not independent of each other. In fact, $\Phi_{l_{i} l_{j} l} \neq 0$ only if the triangle inequality $\left|l_{i}-l_{j}\right| \leq l \leq l_{i}+l_{j}$ is satisfied. ${ }^{48}$ Because of this selection rule and because we intend to restrict the discussion to the leading terms in the expansion Eq. (3.5) for the sake of simplicity, only terms proportional to $\Phi_{000}, \Phi_{220}, \Phi_{221}$, $\Phi_{202}$ and $\Phi_{022}$ will be considered. Of these, $\Phi_{000}=(4 \pi)^{-3 / 2}$ contributes only to $\varphi_{\text {iso }}$. In the context of this study, $\Phi_{221}$ is of particular interest because it accounts for the handedness of the interaction between a pair of mesogens in leading order. ${ }^{50}$

Based upon this truncation of the expansion in Eq. (3.5) and grouping individual terms according to the criteria of Giura and Schoen, ${ }^{48}$ we cast $\varphi_{\text {anis }}$ as

$$
\varphi_{\text {anis }}\left(\boldsymbol{r}_{i j}, \omega_{i}, \omega_{j}\right)=-4 \varepsilon_{\mathrm{mm}}\left(\frac{\sigma}{r_{i j}}\right)^{6} \Psi\left(\widehat{\boldsymbol{r}}_{i j}, \omega_{i}, \omega_{j}\right),
$$

where the anisotropy function is given by

$$
\begin{aligned}
\Psi\left(\widehat{\boldsymbol{r}}_{i j}, \omega_{i}, \omega_{j}\right)= & 5 \varepsilon_{1} P_{2}\left[\widehat{\boldsymbol{u}}\left(\omega_{i}\right) \cdot \widehat{\boldsymbol{u}}\left(\omega_{j}\right)\right] \\
& +5 \varepsilon_{2}\left\{P_{2}\left[\widehat{\boldsymbol{r}}_{i j} \cdot \widehat{\boldsymbol{u}}\left(\omega_{i}\right)\right]+P_{2}\left[\widehat{\boldsymbol{r}}_{i j} \cdot \widehat{\boldsymbol{u}}\left(\omega_{j}\right)\right]\right\} \\
& +\varepsilon_{3}\left[\widehat{\boldsymbol{u}}\left(\omega_{i}\right) \times \widehat{\boldsymbol{u}}\left(\omega_{j}\right)\right] \cdot \widehat{\boldsymbol{r}}_{i j}\left[\widehat{\boldsymbol{u}}\left(\omega_{i}\right) \cdot \widehat{\boldsymbol{u}}\left(\omega_{j}\right)\right] .
\end{aligned}
$$

Throughout this work, we fix the (dimensionless) anisotropy parameters $2 \varepsilon_{1}=-\varepsilon_{2}=0.08$. Closer scrutiny reveals that the first term on the right side of Eq. (3.7) describes the orientation dependence of interactions in the Mayer-Saupe mean-field model of a nematic liquid crystal. ${ }^{45,51,52}$ The next two terms are a correction describing the orientation dependence of the anisotropic attractions with enhanced sophistication whereas the last term is related to $\Phi_{221}$. 
Mathematically speaking, this term is a pseudo-scalar that changes sign if $\omega \rightarrow \omega^{\prime}=-\omega$ on account of the parity rule for spherical harmonics, ${ }^{49}$ that is, if we exchange the center-ofmass positions of mesogens $i$ and $j$ (i.e., for $\widehat{\boldsymbol{r}}_{i j} \rightarrow \widehat{\boldsymbol{r}}_{i j}^{\prime}=-\widehat{\boldsymbol{r}}_{i j}$ ). Notice also that this term remains invariant if $\omega_{i}$ or $\omega_{j}$ are replaced by $-\omega_{i}$ or $-\omega_{j}$ thus preserving the head-tail symmetry of the mesogens. The sign of the chirality coupling constant $\varepsilon_{3}$ controls the handedness of the interaction whereas $\left|\varepsilon_{3}\right|$ controls the pitch $p$ of helical structures that may form provided $\left|\varepsilon_{3}\right|$ is sufficiently large (see Sec. IV B below). The introduction of chirality in our model follows in spirit earlier simulation work by Memmer et al. ${ }^{43,53}$ who modified the achiral Gay-Berne model to allow for the formation of helicoidal (bulk) phases.

We note in passing that Hess and $\mathrm{Su}^{54}$ were the first to suggest the achiral form of the anisotropy function $\left(\varepsilon_{3}=0\right)$ in Eq. (3.7) for a liquid crystal that exhibits a nematic besides the more conventional isotropic liquid and gaseous phases. However, unlike Giura and Schoen, ${ }^{48}$ Hess and Su base their derivation of $\Psi$ on an expansion of $\varphi_{\text {anis }}$ in terms of irreducible Cartesian tensors.

\section{B. Confinement to mesoscopic channels}

The chiral model liquid crystal introduced in Sec. III A is now placed in mesoscopic channels with cross sections $s_{\mathrm{y}}$ and $s_{\mathrm{Z}}$ in the $y$ - and $z$-directions; in the $x$-direction, the channels are supposed to be infinitely long which we realize by applying periodic boundary conditions across the $y-z$ planes located at $x= \pm s_{\mathrm{x}} / 2$ on account of the short-range character of $\varphi_{\mathrm{mm}}$.

From Fig. 1, one can identify three different zones in the mesochannels as far as the interaction between a mesogen and the mesochannel's wall is concerned. In zone I, the mesogen interacts with both the top/bottom and left/right straight portions of the wall. In zone II, a mesogen interacts either with the upper or lower or with the left or right wall. Finally, in zone III, a mesogen interacts with the nearest point on the mesochannel's wall.

Because of these three different zones, the mesogen-wall configurational potential energy [see Eq. (3.1)] can be cast as

$$
\begin{aligned}
\Phi_{\mathrm{mw}}(\boldsymbol{R}, \boldsymbol{\Omega})= & \sum_{i=1}^{N}\left[\varphi_{\mathrm{mw}}\left(\Delta y_{i}, \boldsymbol{r}_{i}, \omega_{i}\right) \Theta\left(\zeta_{i, \mathrm{y}}\right)\right. \\
& +\varphi_{\mathrm{mw}}\left(\Delta z_{i}, \boldsymbol{r}_{i}, \omega_{i}\right) \Theta\left(\zeta_{i, \mathrm{z}}\right) \\
& \left.+\varphi_{\mathrm{mw}}\left(\Delta d_{i}, \boldsymbol{r}_{i}, \omega_{i}\right) \Theta\left(-\zeta_{i, \mathrm{y}}\right) \Theta\left(-\zeta_{i, \mathrm{z}}\right)\right],
\end{aligned}
$$

where $\Theta$ denotes the Heaviside function, $\zeta_{i, \alpha} \equiv\left|\alpha_{i}\right|-s_{\alpha} / 2$ $+R, \Delta \alpha_{i}=\alpha_{i}-\operatorname{sgn}\left(\alpha_{i}\right) s_{\alpha} / 2(\alpha=\mathrm{y}$ or $\mathrm{z})$,

$$
\operatorname{sgn}\left(\alpha_{i}\right)=\left\{\begin{array}{rr}
-1, & \alpha_{i}<0 \\
0, & \alpha_{i}=0 \\
+1, & \alpha_{i}>0
\end{array}\right.
$$

is the signum function, and $R$ is the radius of an arc of the wall (see Fig. 1). This implies that $2 R \leq \min \left(s_{\mathrm{y}}, s_{\mathrm{z}}\right)$. Because of this definition and our choice of the origin of the Cartesian coordinate system at the center of the simulation cell, $\Delta \alpha_{i} \geq 0$ and $\Delta d_{i} \geq 0$ whereas $\zeta_{i, \alpha}$ may be positive, negative, or vanish.

With the aid of the sketch shown in Fig. 1, it is clear that the first two terms on the right side of Eq. (3.8) represent the

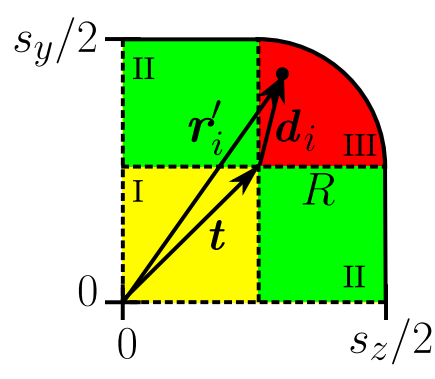

FIG. 1. Sketch of the model system in the $y-z$ plane where the coordinate system has been chosen such that $-s_{\mathrm{y}} / 2 \leq y \leq s_{\mathrm{y}} / 2$ and $-s_{\mathrm{z}} / 2 \leq z \leq s_{\mathrm{z}} / 2$. For symmetry reasons, only the upper right quadrant is shown. In zones I-III separated from each other by dashed lines, a mesogen located at a point $\boldsymbol{r}_{i}^{\prime}$ in the $y-z$ plane interacts with different portions of the wall of the mesochannel (here shown for a mesogen located in zone III, see text). The position of the wall is demarcated by the solid line where $R$ denotes the radius of curvature of the outer surface of zone III such that $\Delta d_{i}=R-\left|d_{i}\right|$ is the shortest distance of a mesogen in zone III from the wall of the mesochannel.

interaction of a mesogen located in one of the zones labelled II; for a mesogen located in zone I, $\Theta\left(\xi_{i, \mathrm{y}}\right)=\Theta\left(\xi_{i, \mathrm{z}}\right)=1$. Hence, quantities $\Delta y_{i}$ and $\Delta z_{i}$ are the minimum distances of a mesogen from the nearest straight portion of the wall of the mesochannel. The third term in Eq. (3.8) accounts for the interaction between a mesogen and the container wall if the mesogen is located somewhere in zone III. Here, $\Delta d_{i}=R-\left|\boldsymbol{d}_{i}\right|$ is the minimum distance of such a mesogen from the container wall. The significance of $\boldsymbol{d}_{i}=\boldsymbol{r}_{i}^{\prime}-\boldsymbol{t}$ is illustrated by the sketch in Fig. 1.

The mesogen-wall interactions are described by the potential function

$\varphi_{\mathrm{mw}}\left(\delta_{i}, \boldsymbol{r}_{i}, \omega_{i}\right)=\varepsilon_{\mathrm{mw}}\left[\frac{2}{5}\left(\frac{\sigma}{\delta_{i}}\right)^{10}-\left(\frac{\sigma}{\delta_{i}}\right)^{4} g\left(\boldsymbol{r}_{i}, \omega_{i}\right)\right]$,

where $\varepsilon_{\mathrm{mw}}=2 \varepsilon$ is the depth of the attractive well of the mesogen-wall potential and $\delta_{i}$ is taken to be $\Delta y_{i}, \Delta z_{i}$, or $\Delta d_{i}$ depending on which of the Heaviside functions (or their product) are equal to one in Eq. (3.8).

The strength of the mesogen-wall interaction has been chosen on the one hand to prevent the confined liquid crystal from freezing in the corners of the mesochannels where the mesogen-wall attraction is strongest. On the other hand, to realize a sufficiently stable alignment of mesogens at the wall, the mesogen-wall attraction must not be too weak. Our choice $\varepsilon_{\mathrm{mw}}=2 \varepsilon$ is a reasonable compromise.

In the actual simulations, we typically find $U_{\mathrm{mm}}$ $=\left\langle\Phi_{\mathrm{mm}}(\boldsymbol{R}, \boldsymbol{\Omega}) / N\right\rangle \simeq-7.00 \varepsilon_{\mathrm{mm}}$ and $U_{\mathrm{mw}}=\left\langle\Phi_{\mathrm{mw}}(\boldsymbol{R}, \boldsymbol{\Omega}) / N\right\rangle$ $\simeq-0.20 \varepsilon_{\mathrm{mm}}$ for the mesogen-mesogen and mesogen-wall perparticle potential energies, respectively, depending somewhat on the exact geometry of the mesochannel. Hence, following the line of arguments presented by de Gennes and Prost (see pp. 113-114 of Ref. 2) this corresponds to the limit of weak anchoring because $U_{\mathrm{mw}} \ll U_{\mathrm{mm}}$ and therefore to an extrapolation length $b$ that is large on a molecular length scale.

The function $0 \leq g\left(\boldsymbol{r}_{i}, \omega_{i}\right) \leq 1$ in Eq. (3.10) is the socalled anchoring function. Depending on $\widehat{\boldsymbol{u}}\left(\omega_{i}\right)$, the anchoring function switches on or off attractive mesogen-wall interactions. This energetic discrimination serves to stabilize specific alignment scenarios. The anchoring function should 
therefore be viewed as a mathematical device to realize specific chemical or mechanical surface preparation as it is frequently employed in experimental systems. A host of different techniques to accomplish specific surface anchoring is known experimentally for quite some time. ${ }^{55,56}$

In this work, we consider three different alignment scenarios. First, if

$$
g\left(\boldsymbol{r}_{i}, \omega_{i}\right)=g_{\perp}\left(\boldsymbol{r}_{i}, \omega_{i}\right)=\left[\widehat{\boldsymbol{u}}\left(\omega_{i}\right) \cdot \widehat{\boldsymbol{s}}\left(\boldsymbol{r}_{i}\right)\right]^{2},
$$

locally homeotropic alignment is favored where $\widehat{\boldsymbol{s}}\left(\boldsymbol{r}_{i}\right)$ is the local surface normal. To realize a locally planar alignment, two options exist. In the first of these, the orientation of a mesogen is orthogonal to both $\widehat{\boldsymbol{s}}\left(\boldsymbol{r}_{i}\right)$ and $\widehat{\boldsymbol{e}}_{\mathrm{x}}$ (i.e., to the $x$-axis). This planar anchoring can be realized by introducing the anchoring function

$$
\begin{aligned}
g\left(\boldsymbol{r}_{i}, \omega_{i}\right)= & g_{\|}\left(\boldsymbol{r}_{i}, \omega_{i}\right)=\left[1-\left|\widehat{\boldsymbol{u}}\left(\omega_{i}\right) \cdot \widehat{\boldsymbol{s}}\left(\boldsymbol{r}_{i}\right)\right|\right]^{2} \\
& \times\left\{\left[\widehat{\boldsymbol{u}}\left(\omega_{i}\right) \cdot \widehat{\boldsymbol{e}}_{\mathrm{y}}\right]^{2}+\left[\widehat{\boldsymbol{u}}\left(\omega_{i}\right) \cdot \widehat{\boldsymbol{e}}_{\mathrm{z}}\right]^{2}\right\} .
\end{aligned}
$$

In Eq. (3.12), the first term on the right side favors planar anchoring whereas the second term allows one to specialize to anchoring of mesogens orthogonal to the $x$-axis (i.e., orthogonal to the symmetry axis of the mesochannel). In addition, we consider directional anchoring in which the energetically favorable alignment of mesogens is still locally planar with the walls of the mesochannel but this time it is also parallel with its symmetry ( $x$-)axis. Hence, for directional anchoring, we choose

$$
g\left(\boldsymbol{r}_{i}, \omega_{i}\right)=g_{\mid}\left(\boldsymbol{r}_{i}, \omega_{i}\right)=\left[1-\left|\widehat{\boldsymbol{u}}\left(\omega_{i}\right) \cdot \widehat{\boldsymbol{s}}\left(\boldsymbol{r}_{i}\right)\right|\right]^{2}\left[\widehat{\boldsymbol{u}}\left(\omega_{i}\right) \cdot \widehat{\boldsymbol{e}}_{\mathrm{x}}\right]^{2}
$$

as the appropriate anchoring function. Notice that both anchoring scenarios represented by Eqs. (3.12) and (3.13) are monostable in the sense of Jérôme. ${ }^{57}$

Last but not least, we can also control the geometry of the mesochannels. This is illustrated by the plots in Fig. 2 for different values of $R$. For vanishing $R$, the mesochannel is rectangular whereas the corners become more rounded as $R$ increases all the way to a cylindrical channel in part (c) of the figure. As anticipated, the minimum of the mesogenwall potential is located in the immediate vicinity of the channel walls. The mesogen-wall potential decays rapidly as one moves away from these walls and remains very weakly attractive as one approaches the mesochannel's midpoint.

\section{RESULTS}

\section{A. Numerical details}

To analyze the local structure of the confined chiral liquid crystal introduced in Sec. III, we perform MC simulations in the grand canonical and in the canonical ensembles. Henceforth, we shall express all quantities in the customary dimensionless (i.e., "reduced") units. We shall express length in units of $\sigma$, energy in units of $\varepsilon$, therefore temperature in units of $\varepsilon / k_{\mathrm{B}}$ ( $k_{\mathrm{B}}$ Boltzmann's constant), and finally pressure in units of $\varepsilon / \sigma^{3}$.

For the bulk liquid crystal, we know from previous work $^{33}$ that for a sufficiently large chirality coupling constant $\left|\varepsilon_{3}\right|=0.25$, our liquid crystal is stable in blue phase II for a temperature $T=0.95$ and a pressure of $P=1.81$. Under these conditions the bulk number density is $\rho \simeq 0.92$. As we shall demonstrate shortly in Sec. IV B, $p$ is proportional to $\left|\varepsilon_{3}\right|^{-1}$. Thus, we can set up the simulation cell such that its linear dimensions are an integer multiple of $p / 2$ to make sure that the helices of blue phase II are not exposed to any spurious strain. We are then in a position to determine the number of mesogens $N$ that our simulation cell should accommodate under these conditions.

For this $N$ and the given $T$ and $P$, we determine the associated chemical potential $\mu \simeq-11.88$ in a corresponding MC simulation in the isothermal-isobaric ensemble by using a properly adjusted version of Widom's test particle method. In this adapted version the fluctuating volume is included in the ensemble average of the Boltzmann factor of the test particle's configurational potential energy. ${ }^{58}$ In a subsequent MC simulation in the grand canonical ensemble, we use this $\mu, T$, and the mean side lengths from the isothermal-isobaric simulation to obtain a mean number density and pressure both of which agree to within a few percent with the input values employed in the isothermal-isobaric simulation. In particular, we monitored the acceptance ratio for creation/deletion of mesogens which always exceeded $5.0 \times 10^{-4}$ so that the results

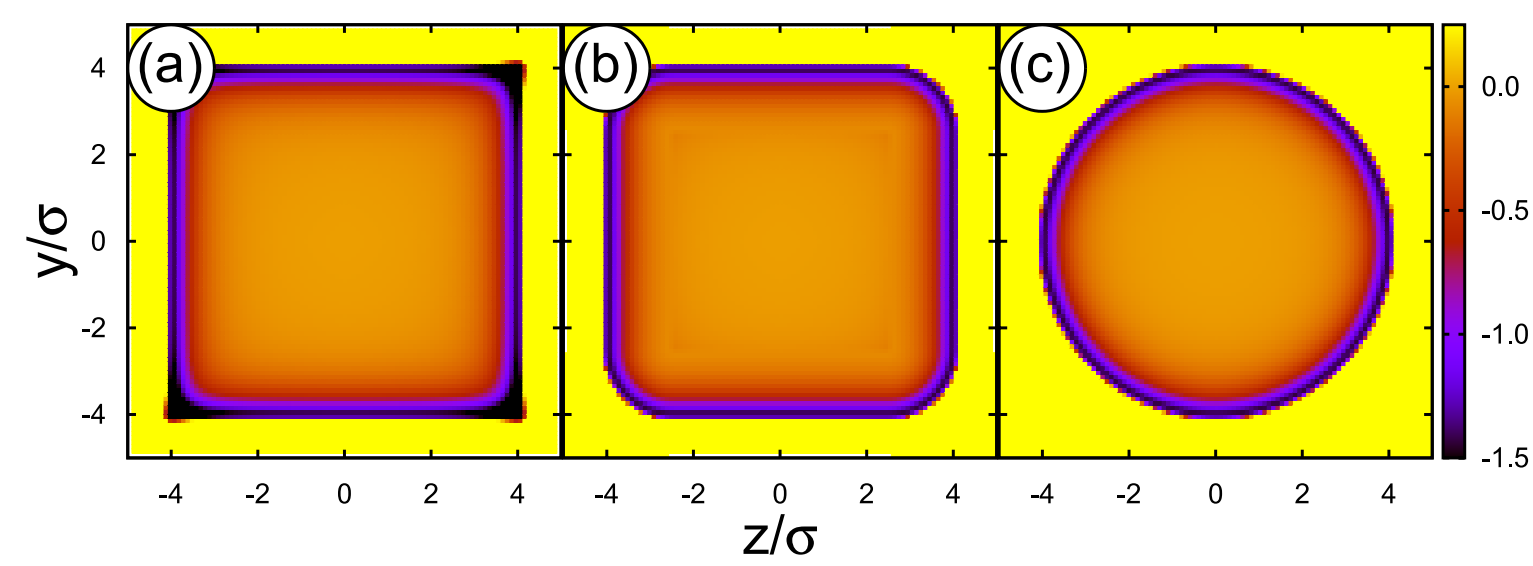

FIG. 2. Plots of the mesogen-wall equipotential surface in the $y-z$ plane generated via Eq. (3.10) for $g\left(\boldsymbol{r}_{i}, \omega_{i}\right)=1$. The attached color bar gives the local value of $\varphi_{\mathrm{mw}}$ for $s_{\mathrm{y}}=s_{\mathrm{z}}=10 \sigma$ illustrating its short-range character. (a) $R / s_{\mathrm{y}}=0$, (b) $R / s_{\mathrm{y}}=0.25$, and (c) $R / s_{\mathrm{y}}=0.50$. 
obtained can safely be considered reliable and the two sets of simulations are mutually consistent with each other.

For all simulations, we use the same $\mu$ and $T$ and adjust the linear dimensions of the simulation box such that in the $x$-direction, $2 s_{\mathrm{x}} / p=k(k \in \mathbb{N})$, where $k$ has been chosen such as to limit the number of mesogens to the range $1.0 \times 10^{4} \leq N$ $\leq 1.5 \times 10^{4}$ and therefore the CPU time needed; side lengths $s_{\alpha}(\alpha=\mathrm{y}$ or $\mathrm{z})$ have been chosen so that a helix of pitch $p$ can be accommodated perfectly or is strained in a controlled way. Notice that in practice, a value of $2 \sigma$ has to be added to $s_{\alpha}$ on account of the excluded volume at the walls. ${ }^{34}$

In MC simulations in the grand canonical ensemble, two events allow one to generate a Markov chain of configurations numerically. In the first of these, it is decided with equal probability whether to displace a mesogen's center of mass or to rotate it around a randomly chosen axis. The outcome of both processes is accepted (or rejected) on the basis of an energy criterion ${ }^{59}$ to make sure that the limiting distribution of configurations in configuration space complies with the probability density of the grand canonical ensemble. During this step of the MC simulations, each of the $N$ mesogens presently in the system is considered sequentially.

During the second step of the MC simulation in the grand canonical ensemble, it is decided with equal probability whether to remove a randomly chosen mesogen from the system or to add a new one at a randomly chosen position and with a randomly chosen orientation. Again, an energy criterion is employed for both processes. ${ }^{59}$ This second step is performed $N^{\prime}$ times where $N^{\prime}$ is the number of mesogens present at the beginning of the second step. Together, the $N+N^{\prime}$ attempts to generate a new configuration are referred to as a "MC cycle" in the grand canonical ensemble; in the canonical ensemble, a MC cycle consist of $N$ attempts to either displace a mesogen's center of mass or to rotate it.

In the grand canonical ensemble, the system is equilibrated for $2.0 \times 10^{4} \mathrm{MC}$ cycles followed by another $1.0 \times 10^{5}$ cycles during which we monitor in particular the mean number of mesogens $\langle N\rangle$ to make sure that the actual number of mesogens $N$ fluctuates around this average value. Once this has been accomplished, we take $N=\langle N\rangle$ as input for a corresponding simulation of $5.0 \times 10^{5}$ cycles in the canonical run where we compute $\mathbf{Q}(\boldsymbol{r})$ via Eq. (2.2) based upon the assumption of equivalence of statistical physical ensembles. We switch to the canonical ensemble to save CPU time; in the grand canonical ensemble, MC simulations are severely slowed down by the attempted creation and deletion of mesogens. Other parameters/techniques employed in the simulations have been chosen/implemented exactly as discussed elsewhere. ${ }^{33}$

\section{B. Bulk system}

We begin our presentation of results with a discussion of the bulk system. In the absence of chirality [i.e., for $\varepsilon_{3}=0$, see Eq. (3.7)] our model is capable of forming gaseous and isotropic and nematic liquidlike phases. ${ }^{48}$ The nematic phase is characterized by a global director $\widehat{\boldsymbol{n}}$ and a scalar nematic order parameter $S$. For $\left|\varepsilon_{3}\right|>0$, more complex ordered phases form that are dominated by helical structures such as a cholesteric phase and blue phases. ${ }^{33}$ These structures can be analyzed quantitatively by determining the local director field $\widehat{\boldsymbol{n}}(\boldsymbol{r})$, the associated order-parameter field $S(\boldsymbol{r})$, and the pitch $p$ of the helix/helices forming under favorable thermodynamic conditions and suitable choices for $\varepsilon_{3} \neq 0$.

In a computer simulation, where for short-range interactions, periodic boundary conditions are applied at the faces of the rectangular simulation cell to minimize surface effects, the formation of helical structures poses a problem in principle. Let $\widehat{\boldsymbol{e}}_{\alpha}(\alpha=\mathrm{x}, \mathrm{y}$, or z) be the direction along which a helix of pitch $p$ evolves. Because of the head-tail symmetry of the mesogens, the helix can be accommodated perfectly if $2 s_{\alpha} / p=m$ where $m \in \mathbb{N}$. Only for integer values of $m$, a helix of a given $p$ corresponds to a minimum in the free energy as has recently been demonstrated by Fukuda and Žumer. ${ }^{60}$ Otherwise, the helix is strained (i.e., compressed or stretched) which may eventually lead to its complete destruction or may cause the direction along which the helix evolves to form some unwanted angle other than $\frac{\pi}{2}$ with the faces of the simulation cell.

Unfortunately, $p$ is a priori unknown. To circumvent the problem with improperly chosen dimensions of the simulation cell, Melle et al. ${ }^{33}$ have placed the liquid crystal between the planar surfaces of a slit-pore with degenerate planar anchoring ${ }^{57}$ of the mesogens. Suppose, the thermodynamic state has been chosen such that a cholesteric phase would form. Then, the presence of the slit-pore walls and the anchoring of mesogens at the substrate guarantees that the cholesteric helix evolves in a direction perpendicular to the substrate plane. If, for the initial choice of $s_{\mathrm{z}}$, the ratio $2 s_{\mathrm{z}} / p$ is not integer but some rational number, an unstrained partial helix will still form because the orientation of mesogens at one wall and that at the other can relax on account of the degenerate planar anchoring conditions. In a sequence of relatively short MC simulations, one can then adjust $s_{\mathrm{Z}}$ such that upon replacing the slit-pore walls again by periodic boundary conditions, a cholesteric helix of an integer half-pitch ending at $z=+s_{z} / 2$ can be continued properly at $z=-s_{z} / 2$. This helix remains stable even without the walls of the slit-pore. This approach works, in principle, also for blue phases consisting of more complex helical structures than the cholesteric phase. ${ }^{33}$

Experimentally, one can control $p$ by considering binary mixtures in which a small amount of chiral material is immersed into an achiral (nematic) host phase. Depending on the concentration of chiral material, a chiral nematic phase forms characterized by some pitch (see p. 284-285 of Ref. 2) This has been demonstrated originally by Adams and Haas ${ }^{61}$ quite some time ago and later by Cladis ${ }^{25}$ and by Kitzerow et $a l .{ }^{30}$ These authors argue that for fixed $T, p^{-1} \propto c$ where $c$ is the concentration of chiral dopant in the binary mixture. Thus, by varying $c$, one gets a handle on $p$. As pointed out later by Wilson and Earl, ${ }^{62}$ the concentration of chiral dopant is proportional to a change in free energy which in turn may be interpreted as a change in the effective strength of interaction between molecules of the chiral mixture component. Hence, in our single-component model of a chiral liquid crystal, one would anticipate $p \propto\left|\varepsilon_{3}\right|^{-1}$. Figure 3 confirms this expectation.

To demonstrate that the above simulation protocol is adequate, we present in Fig. 4 a "snapshot" of a configuration 


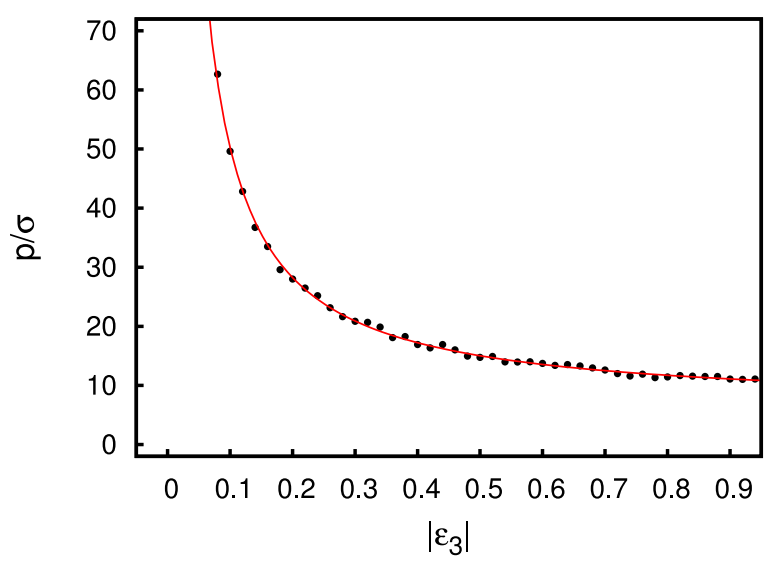

FIG. 3. Plot of the reduced pitch $p / \sigma$ as a function of the chiral coupling constant $\left|\varepsilon_{3}\right|(\bullet)$ obtained in MC simulations ${ }^{33}$ together with a fit of $p$ $=a / \varepsilon_{3}+b$ (red solid line) (see text) where $a \simeq 4.41$ and $b \simeq 6.20$ have been obtained from a least squares fit to the simulation data.

as it was generated as part of the Markov chain in the grand canonical ensemble. A comparison with Fig. 3 reveals that the value of the coupling strength $\left|\varepsilon_{3}\right|$ corresponds to the strong coupling regime where $p$ begins to level off and approaches a nearly constant value. The color code in Fig. 4 has been chosen such that mesogens oriented along the line of vision are colored in blue whereas mesogens lying in the paper plane have been colored red.

As one can see from Fig. 4, four areas can be identified that form a nearly perfect array of fourfold symmetry. These areas extend into the paper plane with the symmetry axis of each area remaining parallel to the line of vision. Focusing on one of the four areas, one notices that at its center mesogens align their longer axes with the line of vision; as one moves away radially from the center to the circumference, the mesogens eventually are lying in the paper plane. This structure comports with that of a double-twist helix. In other words, we obtain

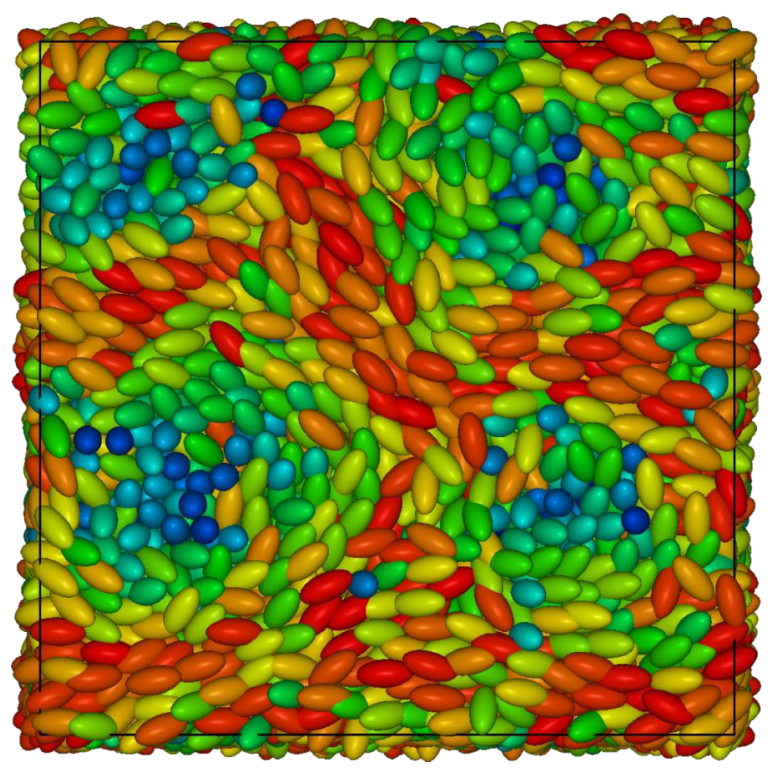

FIG. 4. "Snapshot" of a configuration generated for $\left|\varepsilon_{3}\right|=0.25 \quad(p=23.8$, see Fig. 3). The aspect ratio of mesogens is greatly exaggerated to enhance the visibility.

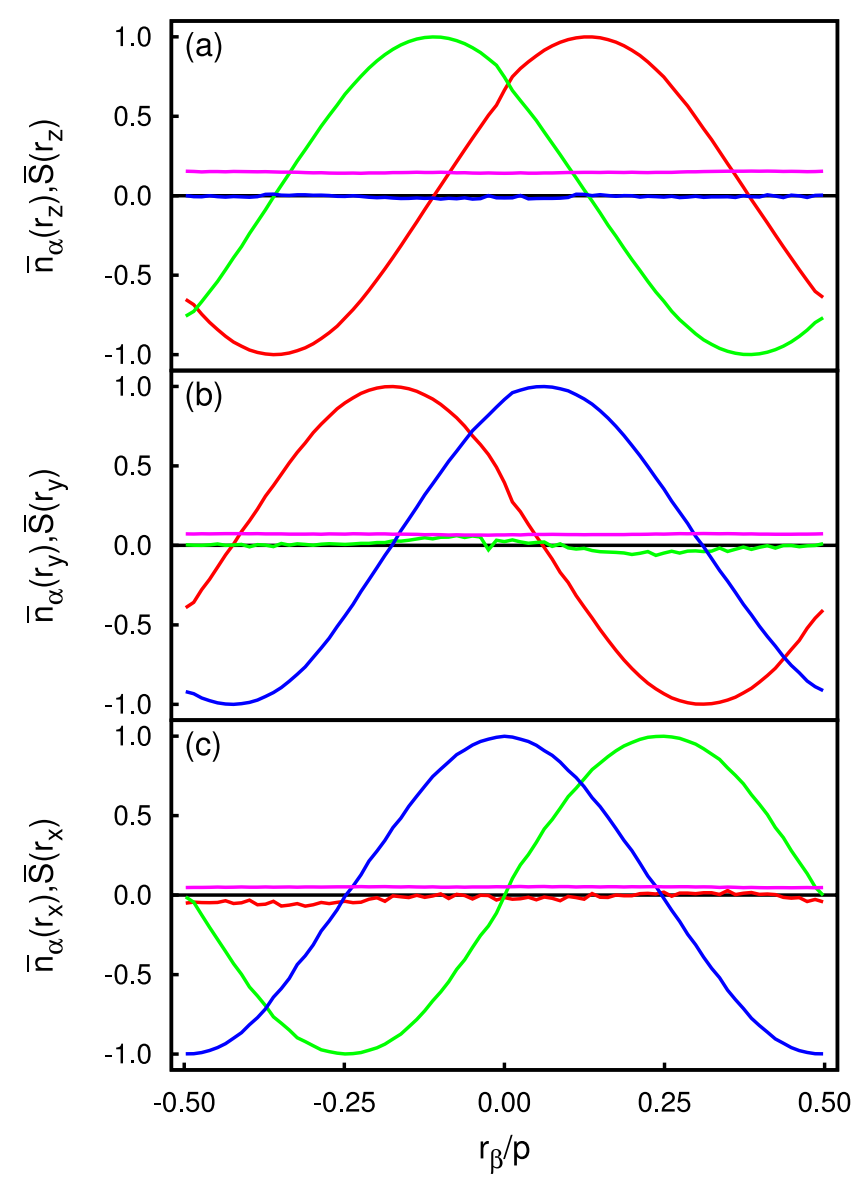

FIG. 5. Plots of components of the nematic director $\bar{n}_{\alpha}\left(r_{\beta}\right)$ and the local nematic order parameter $S\left(r_{\beta}\right)$ (magenta solid line) as functions of specific lines of vision $r_{\beta}=\boldsymbol{r} \cdot \widehat{\boldsymbol{e}}_{\beta}$ where $\alpha=\mathrm{x}$ (red solid line), $\alpha=\mathrm{y}$ (green solid line), and $\alpha=\mathrm{z}$ (blue solid line); (a) $\beta=\mathrm{z}$, (b) $\beta=\mathrm{y}$, and (c) $\beta=\mathrm{x}$.

a simple-cubic array of double-twist helices characteristic of blue phase II. ${ }^{2}$

If one analyzes the structure shown in Fig. 4 more quantitatively in terms of $\widehat{\boldsymbol{n}}(\boldsymbol{r})$ and $S(\boldsymbol{r})$, plots of these quantities in Fig. 5 are indeed indicative of the highly symmetric structure just described. For example, in Figs. 5(a)-5(c), we show that always the component of $\overline{\widehat{\boldsymbol{n}}}\left(r_{\beta}\right)$ along the line of vision $r_{\beta}=\boldsymbol{r} \cdot \widehat{\boldsymbol{e}}_{\beta}(\beta=\mathrm{x}, \mathrm{y}$, or $\mathrm{z})$ approximately vanishes whereas the remaining other two components show a very regular sinusoidal structure. We use the overbar to indicate that $\widehat{\widehat{\boldsymbol{n}}}\left(r_{\beta}\right)$ has been averaged over the plane orthogonal to $r_{\beta}$. These sinusoidal curves are shifted relative to one another by about $\frac{\pi}{2}$. Hence, plots in each panel of Fig. 5 signal the formation of helices evolving in planes orthogonal to $r_{\beta}$.

Nevertheless, the overall nematic order in each of the planes orthogonal to $r_{\beta}$ remains small and nearly vanishes as plots of $\bar{S}\left(r_{\beta}\right)$ in Figs. 5(a)-5(c) reveal. This is consistent with the snapshot presented in Fig. 4. It shows that despite the existence of highly ordered regions in each plane, the overall order obtained as an average of the local nematic order parameter over planes is small or vanishes for each individual plane orthogonal to $r_{\beta}$. Hence, we are dealing with doubletwist helices arranged such that along each line of vision a structure of fourfold symmetry is obtained. These features clearly point to a stable blue phase II. 

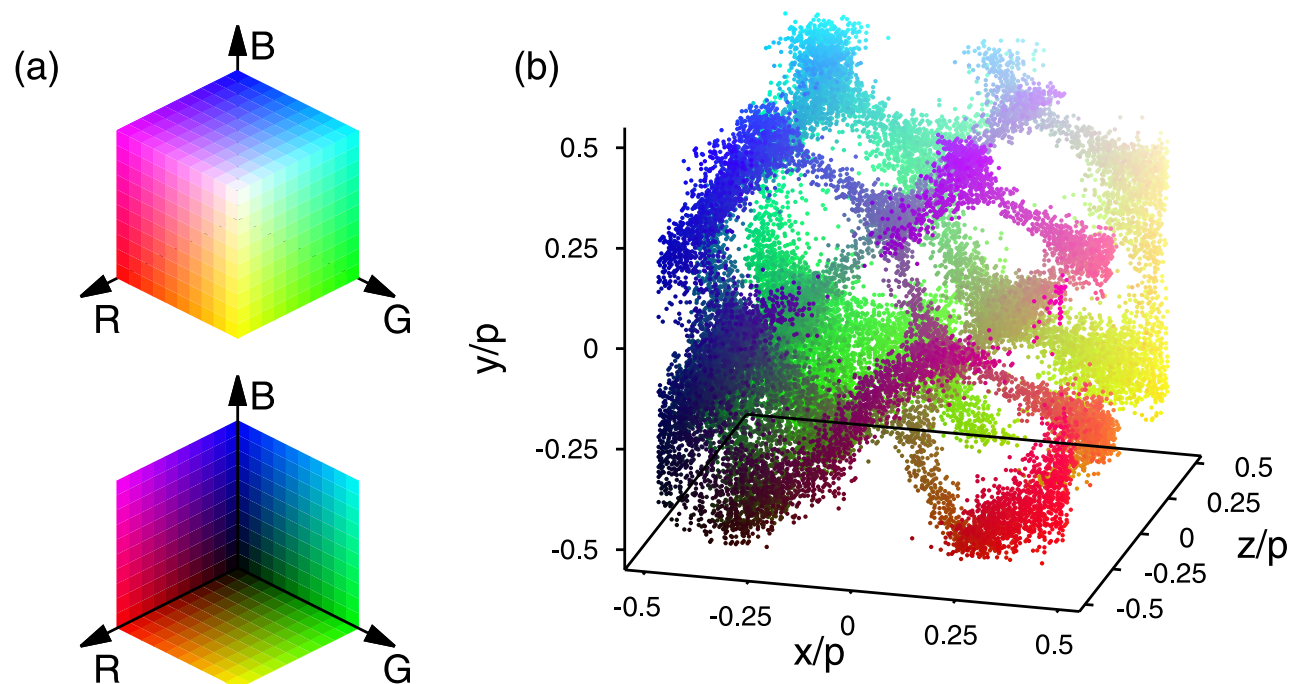

FIG. 6. (a) Color cube to illustrate the spatial variation of color where $\widehat{\boldsymbol{e}}_{\mathrm{R}} \cdot \widehat{\boldsymbol{e}}_{\mathrm{X}}=\widehat{\boldsymbol{e}}_{\mathrm{B}} \cdot \widehat{\boldsymbol{e}}_{\mathrm{y}}=\widehat{\boldsymbol{e}}_{\mathrm{G}} \cdot \widehat{\boldsymbol{e}}_{\mathrm{Z}}=1$ where $\widehat{\boldsymbol{e}}_{\mathrm{R}}, \widehat{\boldsymbol{e}}_{\mathrm{B}}$, and $\widehat{\boldsymbol{e}}_{\mathrm{G}}$ are unit vectors defining the axes used for the color cubes. (b) Disclination lines $\ell$ for blue phase II.

Further evidence for this conclusion is provided by an analysis of disclination lines $\ell$. Because the spatial structure of disclination lines is complex and difficult to visualize, we have developed a color code [see Fig. 6(a)] where a different color is assigned according to the specific point on $\ell$. An inspection of 6(b) shows that under the present conditions, disclination lines form a complex structured three-dimensional network. Most notably, however, is the clear tetrahedral symmetry of the arrangement of these lines. This tetrahedral arrangement of disclination lines is what one would indeed expect for a blue phase II as has been demonstrated earlier by Ravnik et al. ${ }^{13}$ within the framework of Landau-de Gennes theory and a little later by Melle et al. by MC simulations. ${ }^{33}$ Hence, together the results presented in this section provide clear evidence for the formation of a blue phase II in our bulk liquid crystal under the thermodynamic conditions chosen and for the model parameters selected.

Because in blue phase II the liquid crystal is ordered locally but disordered globally, one expects this order to decay monotonically for nonvanishing chirality as the distance between locally ordered regions increases. In the limit of vanishing chirality, however, one is confronted with an ordinary nematic phase which, under the present thermodynamic conditions, is characterized by a global nematic order parameter $S=0.620$. For the nematic phase, one therefore anticipates from Eq. (2.6), $\lim _{r_{\|} \rightarrow \infty} g_{2}\left(r_{\|}\right)=S^{2}$. Plots in Fig. 7 confirm both expectations. In the simulations, we obtain the curves as histograms using cylindrical shells of radius 0.5 and thickness $\Delta r_{\|}=0.05$ [see Eq. (2.6)].

In both curves in Fig. 7, one also notices weak oscillations at smaller $r_{\|}$which disappear for $r_{\|} \gtrsim 5.00$. The oscillations reflect spatial correlations superimposed onto the orientational ones. Once these superimposed spatial correlations have nearly vanished and assuming an exponential decay proportional to $\exp \left(-r_{\|} / \xi\right)$ for larger $r_{\|}$, we get a correlation length which is infinite in the case of the achiral, nematic phase whereas in the blue phase, we obtain $\xi \simeq 2.70$ indicating that nematic correlations are short range for the chiral bulk fluid at $\left|\varepsilon_{3}\right|=0.25$ as one would expect. Taking $\xi$ as a characteristic length scale in our system, $p$ is roughly an order of magnitude larger than $\xi$. Hence, we conclude that the helical structures at the focus of this work are truly supramolecular compared with the range of orientational correlations.

\section{Confinement effects}

If we now bring the bulk liquid crystal into confinement, the pitch as a characteristic length scale will have to compete with the linear dimensions of the confining mesochannel (see Fig. 1). In the following, we always choose $\widehat{\boldsymbol{e}}_{\mathrm{x}}$ as the line of vision. Along $\widehat{\boldsymbol{e}}_{\mathrm{x}}$, we analyze components of $\widehat{\widehat{\boldsymbol{n}}}(x)$ and $\bar{S}(x)$. In addition, we consider $\widehat{\boldsymbol{n}}\left(\boldsymbol{r}^{\prime}\right)$ and $S\left(\boldsymbol{r}^{\prime}\right)$ where $\boldsymbol{r}^{\prime}$ is a twodimensional vector in the $y-z$ plane at a given $x$.

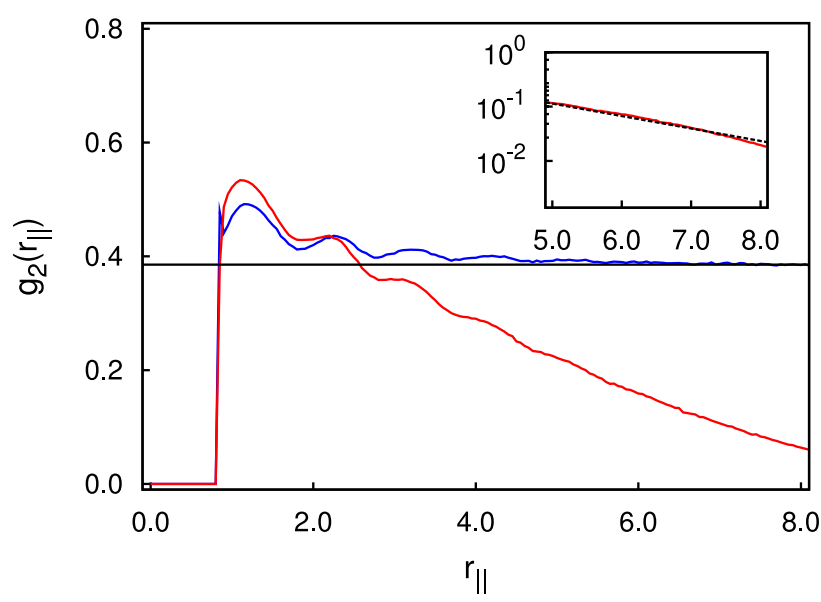

FIG. 7. Plots of the nematic correlation function $g_{2}\left(r_{\|}\right)$defined in Eq. (2.6) where $\varepsilon_{3}=0.00$ (blue solid line ) and $\left|\varepsilon_{3}\right|=0.25$ (red solid line). The horizontal line (black solid line) corresponds to $S^{2}$. The inset is an enlargement of the plot for $\left|\varepsilon_{3}\right|=0.25$ where we also show the function $a \exp \left(-r_{\|} / \xi\right)(---)$ fitted to the simulation data and taking $a$ and the nematic correlation length $\xi$ as fit parameters (see text). 
(a)

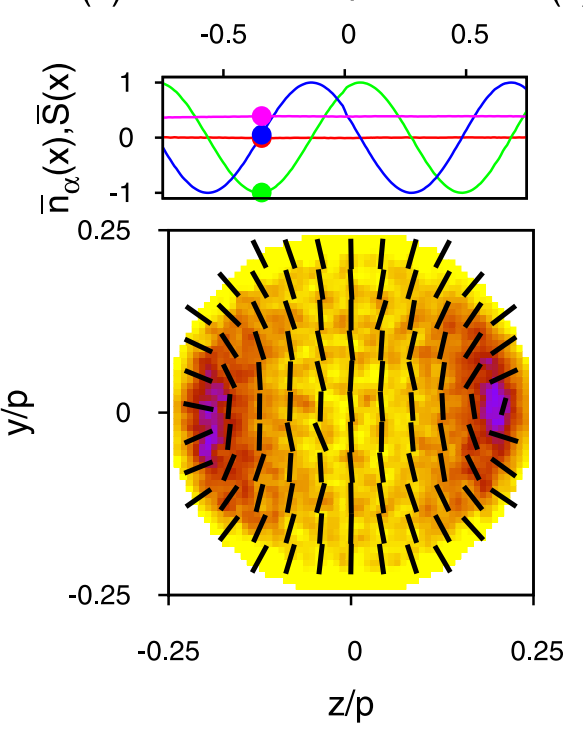

(b)
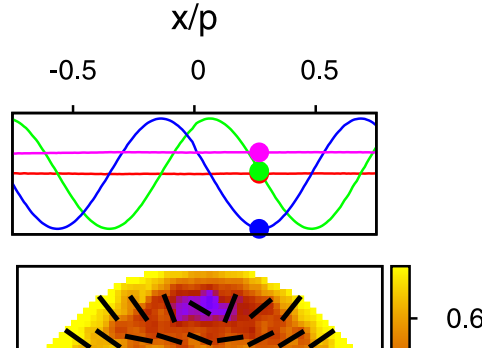
0

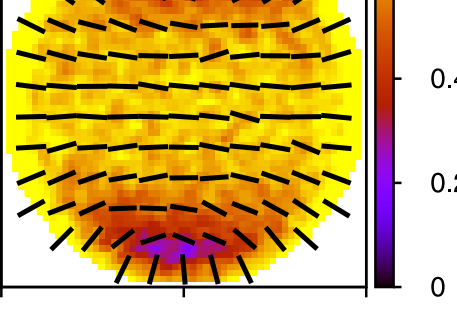

$-0.25$

0

0.25

$z / p$

FIG. 8. (Upper panels) Variation of components of the local director field $\bar{n}_{\alpha}(x)$ along the $x$-axis of a mesochannel for $R / s_{\mathrm{y}}=0.50 ; \alpha=\mathrm{x}$ (red solid line), $\alpha=\mathrm{y}$ (green solid line), and $\alpha=\mathrm{z}$ (blue solid line). Also shown is the local nematic order parameter $\bar{S}(x)$ (magenta solid line). Dots, colored the same way as the above lines, indicate the actual position along the line of vision. (Lower panels) Plot of $\widehat{\boldsymbol{n}}\left(\boldsymbol{r}^{\prime}\right)$ (dashes) for given $x$ as in the upper panel. Values of $S\left(r^{\prime}\right)$ correspond to the attached color bar.

\section{Homeotropic anchoring}

We begin the discussion withone of the simplest cases in Fig. 8 for a mesochannel of width $s_{\mathrm{y}} / p=s_{\mathrm{z}} / p=0.50$ and $R / s_{\mathrm{y}}=0.50$. For these parameters, the mesochannel is cylindrical. In the upper panels of Fig. 8, we display plots of components of the director field $\overline{\widehat{\boldsymbol{n}}}(x)$ along the cylinder's symmetry axis. From these plots, it is evident that $\bar{n}_{\mathrm{x}}(x) \simeq 0$ and that $\bar{n}_{\mathrm{y}}(x)$ and $\bar{n}_{\mathrm{z}}(x)$ can be described by sine functions shifted with respect to each other by a phase of $\frac{\pi}{2}$. Hence, the director field rotates in the $y-z$ plane thus forming a singletwist helix that evolves along the $x$-axis.

In addition, we present the local nematic order parameter $\bar{S}(x)$ in the upper panels of Fig. 8. The nematic order parameter assumes a nearly constant, relatively high value $\bar{S}(x) \simeq 0.50$ indicating that the overall nematic order across each of the $y-z$ planes along the $x$-axis is quite large. In summary, $\overline{\widehat{\boldsymbol{n}}}(x)$ and $\bar{S}(x)$ indicate that inside the cylindrical mesochannel a phase has formed which is reminiscent of a cholesteric phase.

However, this phase is unique (and different from a cholesteric phase in the bulk) in that the director field is not entirely uniform across any $y-z$ plane as one can see from the plot displayed in the lower panels of Fig. 8. Nevertheless, $\widehat{\boldsymbol{n}}\left(\boldsymbol{r}^{\prime}\right)$ is relatively uniform in a fairly large central region. In this region, $S\left(\boldsymbol{r}^{\prime}\right)$ is quite substantial.
Towards the walls of the mesochannel, one notices from Fig. 8 that $\widehat{\boldsymbol{n}}\left(\boldsymbol{r}^{\prime}\right)$ has to bend more and more to accommodate the local homeotropic anchoring of the mesogens directly at the container walls. As a result, $S\left(\boldsymbol{r}^{\prime}\right)$ goes down up to a point where the local nematic order suddenly drops because further bending of $\widehat{\boldsymbol{n}}\left(\boldsymbol{r}^{\prime}\right)$ would cost too much free energy. Instead, two defects are forming in the vicinity of the wall. The director field and defect structure presented in the lower panels of Fig. 8 are well-known for nematic phases confined to mesoscopic cylinders. ${ }^{25,28,63}$ In Ref. 63, however, the director field $\widehat{\boldsymbol{n}}\left(\boldsymbol{r}^{\prime}\right)$ is constant between successive planes along the $x$-axis whereas here it rotates.

Given the fact that $s_{\mathrm{y}} / p=s_{\mathrm{z}} / p=0.50$, the mesochannel would, in principle, be capable of accommodating a single double-twist helix. As we demonstrated in Sec. IV B, doubletwist helices are structures that are stable for our current choice of the chiral coupling constant $\left|\varepsilon_{3}\right|$. Nonetheless, double-twist helices cannot form in the present cylindrical mesochannels with locally homeotropic anchoring because the impact of the walls is too strong given the diameter of the mesoscopic cylinders.

Moreover, regions of low nematic order visible in the plots in the lower panels of Fig. 8 are actually part of threedimensional disclination lines $\ell$ whose evolution in space we illustrate by the plot in Fig. 9(a). From that plot, one (a)

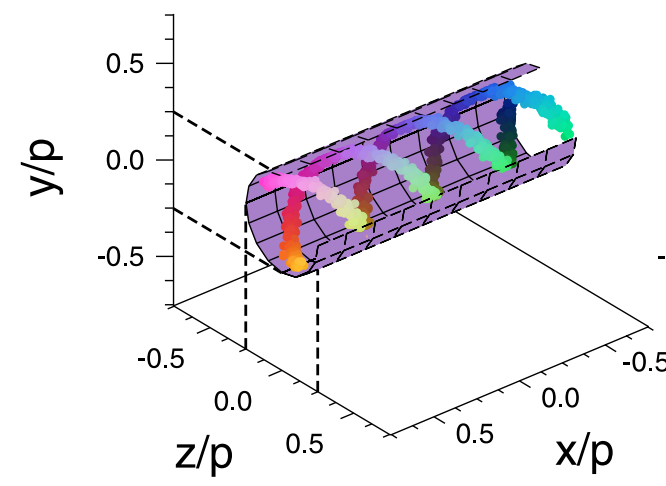

(b)

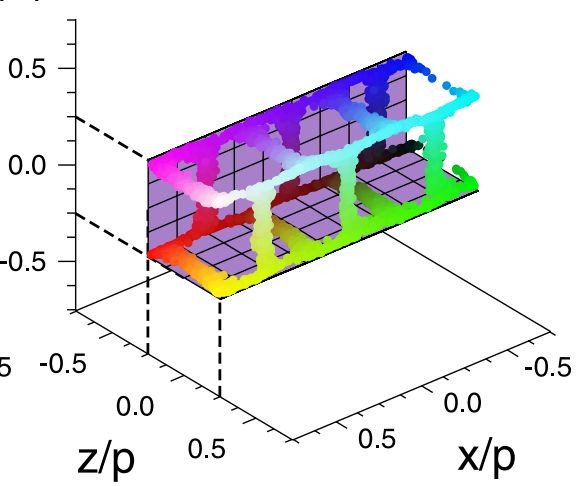

FIG. 9. Three-dimensional representation of disclination lines $\ell$. To enhance the visibility the lines have been colored according to the color cube shown in Fig. 6(a). The mesochannels have been cut open so that one can look inside. Note that this also eliminates part of $\ell$ from the plot. (a) $R / s_{\mathrm{y}}=0.50 s_{\mathrm{y}} / p$ $=s_{\mathrm{z}} / p=0.50,(\mathrm{~b})$ as (a), but for $R / s_{\mathrm{y}}$ $=0.00$. 
(a)

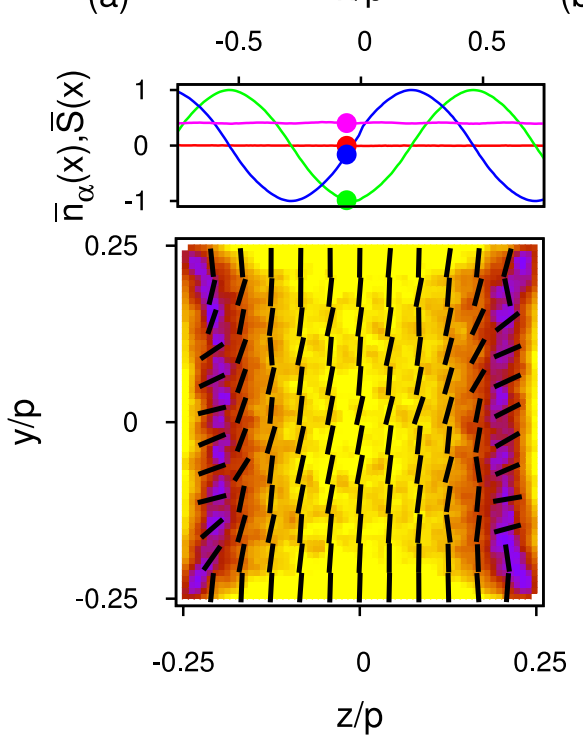

(b)

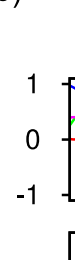

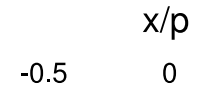

$-0.5$
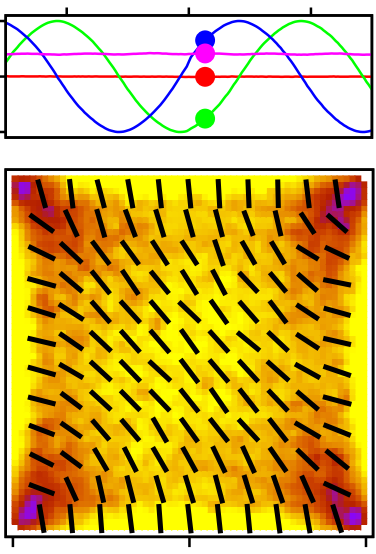

$-0.25$ $x / p$

0.5

(c)

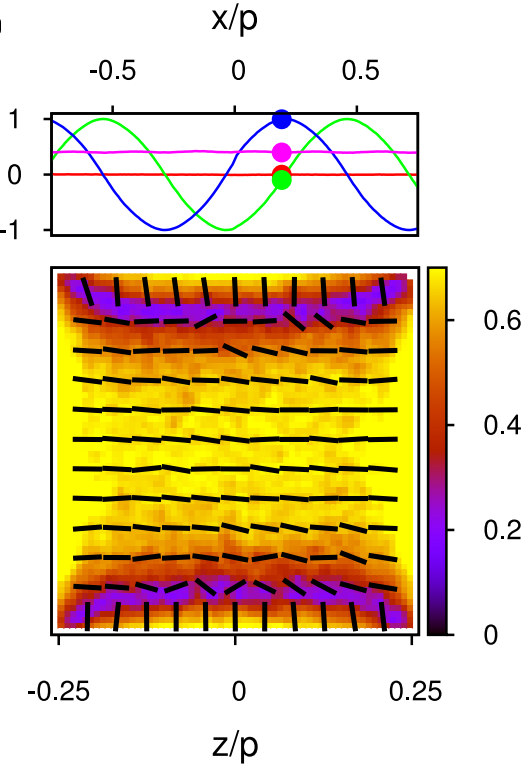

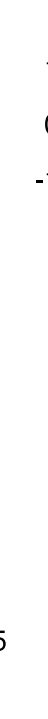

0

$\mathrm{z} / \mathrm{p}$

FIG. 10. As Fig. 8, but for $R / s_{\mathrm{y}}=0.00$. Lower panels (a)-(c) show the local director fields $\widehat{\boldsymbol{n}}\left(\boldsymbol{r}^{\prime}\right)$ and the local nematic order parameter $S\left(\boldsymbol{r}^{\prime}\right)$ (see attached color bar for different positions along the line of vision indicated by the colored dots in the corresponding upper panels).

realizes that, similar to the director field (see Fig. 8), two separate disclination lines exist forming helical structures and evolving in the $x$-direction. These lines never intersect. This disclination-line topology has already been reported by Fukuda and Žumer. ${ }^{31}$ However, in their case, different boundary conditions have been used. This implies that the origin of the defect topology illustrated by the plot in Fig. 9(a) is different from that in work of Fukuda and Žumer.

Changing now $R / s_{\mathrm{y}}$ to 0.00 while maintaining the size of the mesochannel's cross section $s_{\mathrm{y}} / p=s_{\mathrm{z}} / p=0.50$ as in Fig. 8, we notice from the plots in the top panels in Fig. 10 that again the director field is forming a single-twist helix evolving along the $x$-direction. This helix is also visible from plots in the lower panels of Fig. 10 where we indicate the direction of $\widehat{\boldsymbol{n}}\left(\boldsymbol{r}^{\prime}\right)$ for three distinct positions along the line of vision.

Again, one sees that regions of low $S\left(\boldsymbol{r}^{\prime}\right)$ exist which change position in phase with the rotation of $\widehat{\boldsymbol{n}}\left(\boldsymbol{r}^{\prime}\right)$. In Figs. 10(a) and 10(c), these defect regions run more or less parallel with either the $y$-or the $z$-axis. For an intermediate case displayed in Fig. 10(b), the extended defect regions reduce to much smaller regions located in all four corners of the rectangular mesochannel.

As before for the cylindrical mesochannel, the full threedimensional variation of the disclination lines is illustrated by the plot in Fig. 9(b). To make contact between the plots in Fig. 9(b) and those presented in Fig. 10, the reader should realize that the situations depicted in Figs. 10(a) and 10(c) correspond to situations in Fig. 9(b) in which the disclination lines connect to walls of the mesochannel along the $y$ - and $z$-directions, respectively. The case depicted in Fig. 10(b) is representative of regions in which $\ell$ in Fig. 9(b) is perpendicular to the $x$-axis. Hence, the topology of $\ell$ can best be described as that of two ladders in the $y-z$ plane that are connected periodically for certain values of $x$.

If one increases the size of the cross section of a cylindrical mesochannel, the structure of the confined liquid crystal illustrated by plots in Fig. 11 changes significantly from that discussed before [see Fig. 8]. Again, the variation of components of $\overline{\widehat{\boldsymbol{n}}}(x)$ displayed in the upper panels of Fig. 11 is the fingerprint of a helical structure evolving along the line of vision. However, compared with $\bar{S}(x)$ in the upper panel of Fig. 8, this quantity appears to be much reduced in Fig. 11 indicating the presence of more extended regions of low nematic order in each individual $y-z$ plane along the line of vision.

In fact, the plots in the lower panels of Fig. 11 reveal a complex structure of the confined liquid crystal. One notices that at the center of the plots in the lower panels of Fig. 11, the small dashes indicating the local direction of $\widehat{\boldsymbol{n}}\left(\boldsymbol{r}^{\prime}\right)$ are becoming shorter and the closer one moves towards the center of the plots. This indicates that in the central region, the local director field has a nonzero component $n_{\mathrm{x}}\left(\boldsymbol{r}^{\prime}\right)$. Hence, the formation of a double-twist helix, which is favored on account of the magnitude of $\left|\varepsilon_{3}\right|$, is possible here. This is because of the wider cross section of the mesochannel which reduces the impact of wall anchoring on the orientation of the mesogens at its center.

As one moves towards the circumference of the central circular region, mesogens are increasingly lying in the $y-z$ plane as one would expect for a double-twist helix (see Fig. 4). If this planar arrangement of mesogens along the circumference of the double-twist helix would be preserved, a closed circular loop of low $S\left(\boldsymbol{r}^{\prime}\right)$ would have to form eventually. Its formation would be caused by the incompatibility between the inplane orientation of mesogens along the circumference and their homeotropic anchoring at the walls of the mesochannel. However, the system can lower its free energy by forming six spatially rather isolated defect regions, three inner ones at the circumference of the double-twist helix, and three outer ones next to the wall of the mesochannel. Both groups of defects form highly symmetric, trigonal structures rotated with respect to each other by an angle of $\frac{\pi}{3}$. All six regions rotate in the $y-z$ plane as plots in Fig. 11 reveal. 
(a)

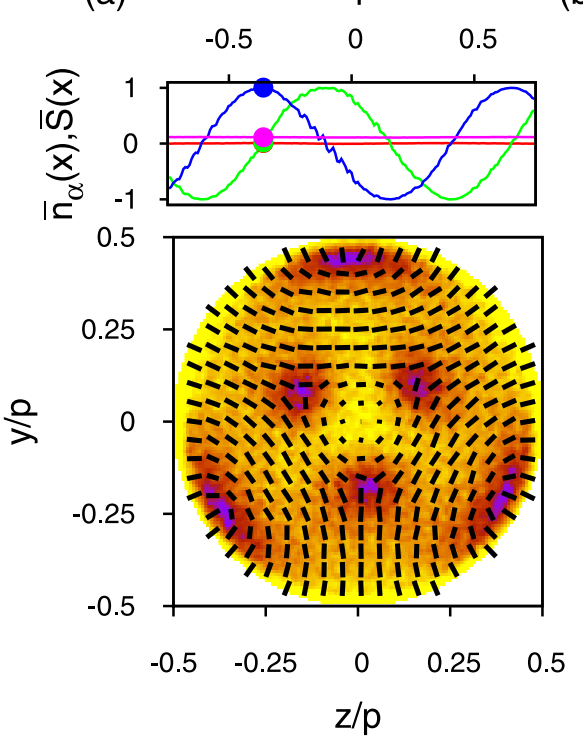

(b)
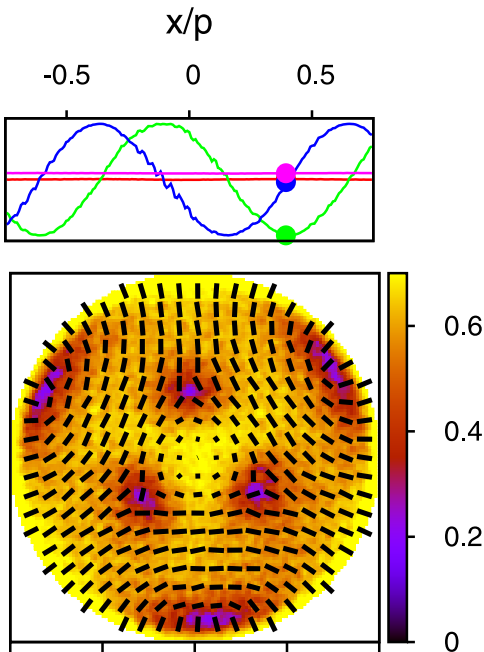

$0.25 \quad 0.5$
FIG. 11. As Fig. 8, but for $s_{\mathrm{y}} / p$ $=s_{\mathrm{z}} / p=1.00$
The full three-dimensional representation of disclination lines $\ell$ is displayed in Fig. 12(a). As one can see from the plot, three helical disclination lines form in the cylindrical mesochannel, which are wrapped around one another without any junctions at which two or all three lines would intersect.

For a rectangular mesochannel of the same sizes of the cross sections, a completely different structure is found. This is illustrated by plots of components of $\overline{\widehat{\boldsymbol{n}}}(x), \bar{S}(x), \widehat{\boldsymbol{n}}\left(\boldsymbol{r}^{\prime}\right)$, and $S\left(\boldsymbol{r}^{\prime}\right)$ in Fig. 13. As before for the cylindrical mesochannel (see Fig. 11), a single double-twist helix forms at the center of the mesochannel as reflected by the decreasing length of dashes. Mesogens at the center of the double-twist helix have their longer axes oriented such that they are orthogonal to the paper plane (see, for example, double-twist helices in Fig. 4). As is characteristic for double-twist helices, this orientation changes as one moves to its circumference where mesogens lie in the paper plane. On account of the in-plane orientation conflicts arise with the homeotropic wall anchoring such that two regions of low $S\left(\boldsymbol{r}^{\prime}\right)$ arise. These regions actually form two helices wrapped around the line of vision as one can see from the plot in Fig. 12(b).

The other feature visible from the plots in Fig. 13 is two regions of low $S\left(\boldsymbol{r}^{\prime}\right)$ running more or less parallel with the upper and lower walls of the mesochannel, respectively. As one moves along the line of vision, the upper/lower configuration of these regions alternates periodically with a left/right configuration in very much the same way as it is observed in Fig. 10. This alternating arrangement can also be seen from the full three-dimensional representation of disclination lines in Fig. 12(b) indicating again a ladder (sub-) structure of $\ell$.

So far in this section, we considered mesochannels characterized by cross sections of equal size in both $y$ - and $z$-directions. The plots in Fig. 14 illustrate the structure of a liquid crystal confined to a mesochannel of a cross section ratio of $s_{\mathrm{y}} / s_{\mathrm{z}}=\frac{3}{4}$. As for the previously discussed cases, components of $\overline{\widehat{\boldsymbol{n}}}(x)$ shown in the upper panels in Fig. 14 indicate that the director field forms some sort of periodically changing structure along the line of vision.

However, in this case, $\bar{n}_{\mathrm{y}}(x)$ and $\bar{n}_{\mathrm{z}}(x)$ can no longer be described by simple trigonometric functions. This is because only the three outer regions of low $S\left(\boldsymbol{r}^{\prime}\right)$ visible in the plot in the lower panels of Fig. 14 turn out to rotate in a counterclockwise fashion as one moves along the line of vision. These rotating outer regions of low $S\left(\boldsymbol{r}^{\prime}\right)$ mark centers of double-twist helices which are incomplete because of their close proximity to the walls. Because, at the center of each (a)

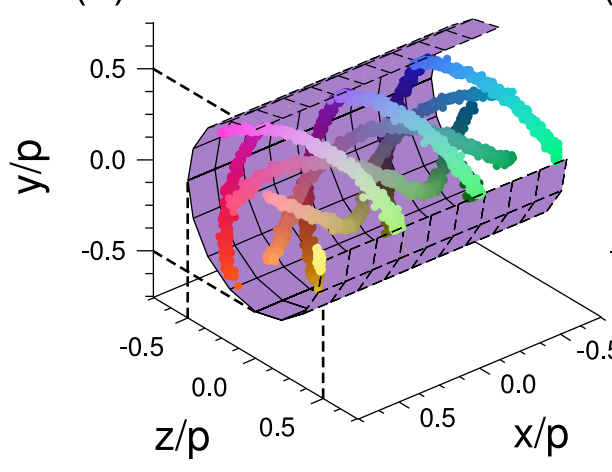

(b)

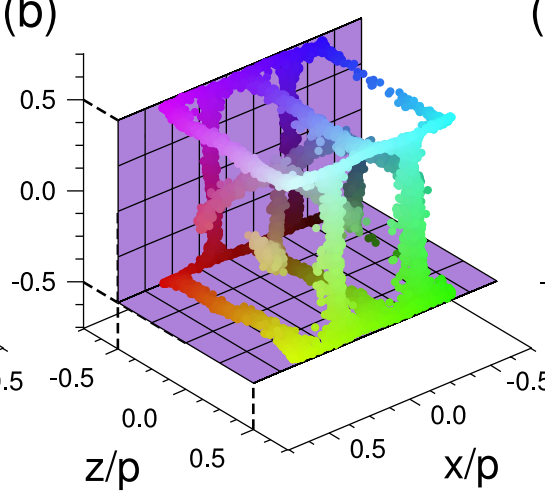

(c)

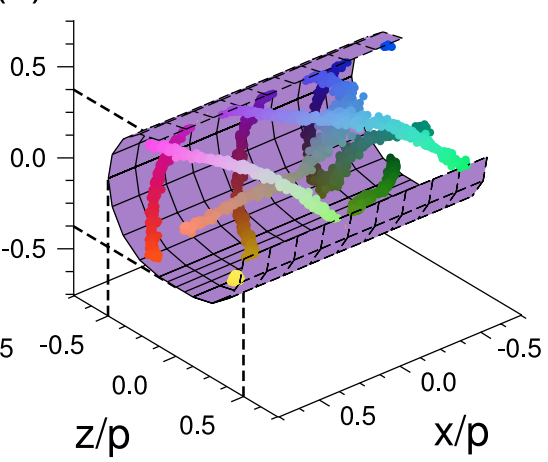

FIG. 12. As Fig. 9, but for (a) $R / s_{\mathrm{y}}=0.50, s_{\mathrm{y}} / p=s_{\mathrm{z}} / p=1.00$, (b) $R / s_{\mathrm{y}}=0.00, s_{\mathrm{y}} / p=s_{\mathrm{z}} / p=1.00$, and (c) $R / s_{\mathrm{y}}=0.50, s_{\mathrm{y}} / p=0.75, s_{\mathrm{z}} / p=1.00$. 
(a)

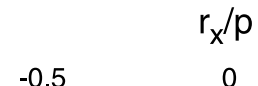

$r_{x} / p$
0

(b)

0.5
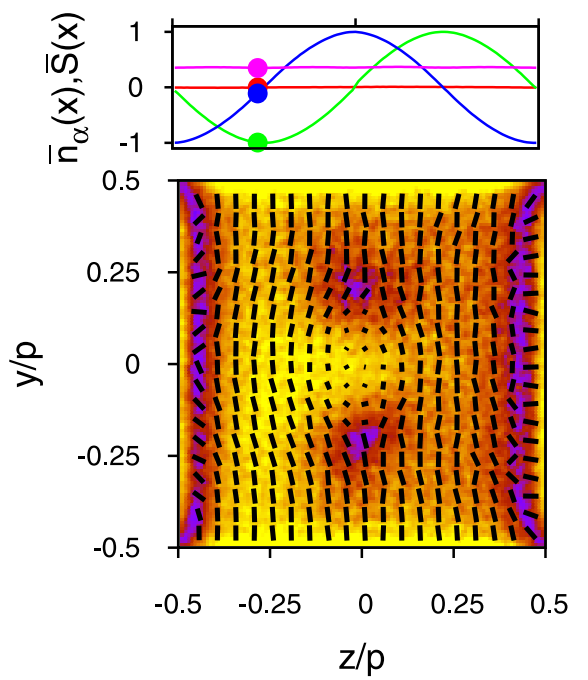

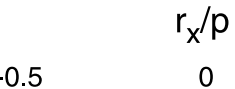

(c)

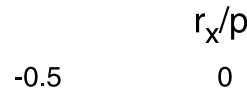

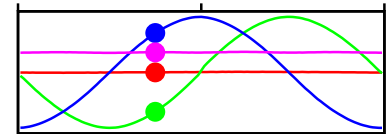

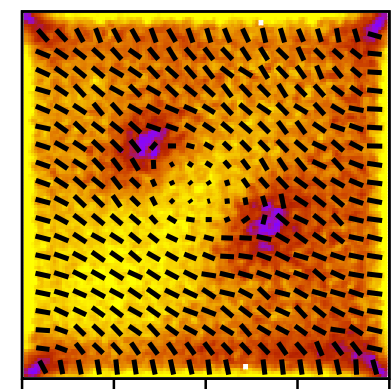

$-0.5$
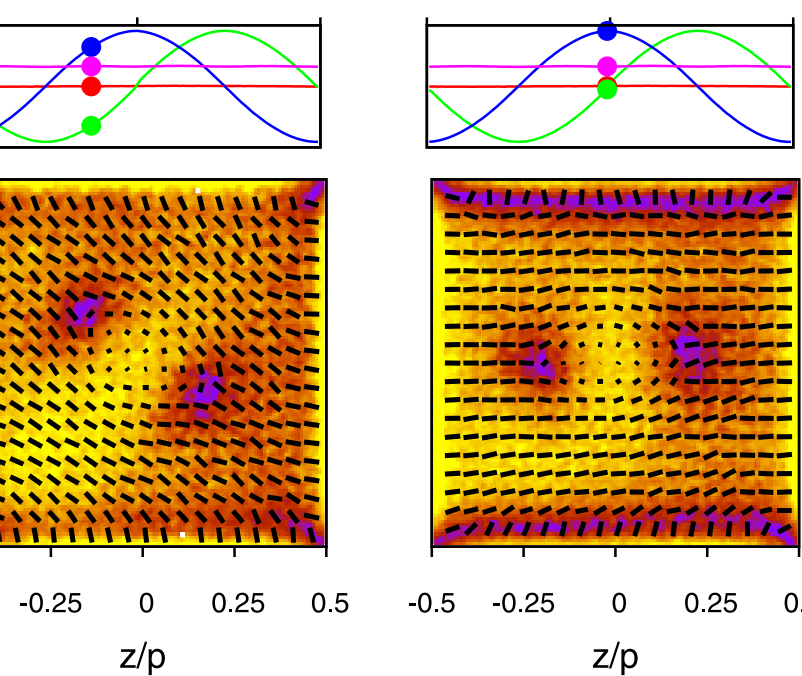

0.5

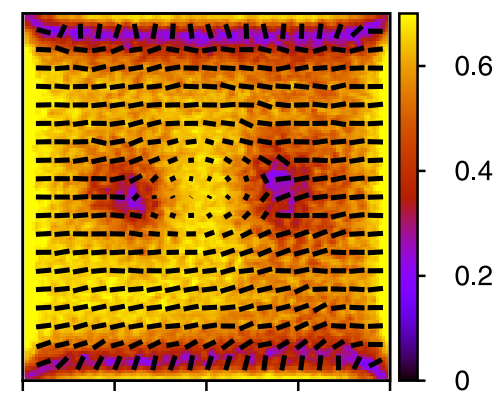

$\begin{array}{rrrrr}-0.5 & -0.25 & 0 & 0.25 & 0.5 \\ & z / p & & \end{array}$

FIG. 13. As Fig. 10, but for $R / s_{\mathrm{y}}=0.00, s_{\mathrm{z}} / p=s_{\mathrm{y}} / p=1.00$.

of the three incomplete double-twist helices, mesogens would have to be aligned separately with the line of vision, a fourth region of low $S\left(\boldsymbol{r}^{\prime}\right)$ is visible in Fig. 14 which arises on account of orientational frustration of mesogens in a region in which the circumferences of all three double-twist helices meet. This region remains stationary and, in fact, forms a straight line as the corresponding three-dimensional plot of disclination lines in Fig. 12(c) clearly indicates. Again, the topology of disclination lines depicted in Figs. 12(c) and 14 has already been observed theoretically in a qualitatively similar fashion (see Fig. 10(c) of Ref. 28).

\section{Planar anchoring}

Compared with the case of homeotropic wall anchoring, planar wall anchoring of the mesogens gives rise to somewhat less spectacular defects. This is an immediate consequence of the fact that for planar anchoring, the orientation of mesogens at the wall is compatible with their in-plane arrangement along the circumference of a double-twist helix.

We consider the case of (locally) monostable planar anchoring first where the anchoring function is given in Eq. (3.12). Take, for example, a cylindrical mesochannel of cross sections $s_{\mathrm{y}} / p=s_{\mathrm{z}} / p=0.50$. This mesochannel is wide enough to accommodate a single double-twist helix where the center of the helix coincides with the center of the mesochannel. At the center of the double-twist helix, mesogens align with the $x$-axis and assume an increasingly planar arrangement in the $y-z$ plane as one moves towards the circumference of the double-twist helix. In this case, the planar anchoring stabilizes a double-twist helix because the orientation of mesogens directly at the walls of the mesochannel is compatible everywhere with their orientation along the circumference of a double-twist helix (cf. Fig. 11). As a consequence, no defects arise under these circumstances.

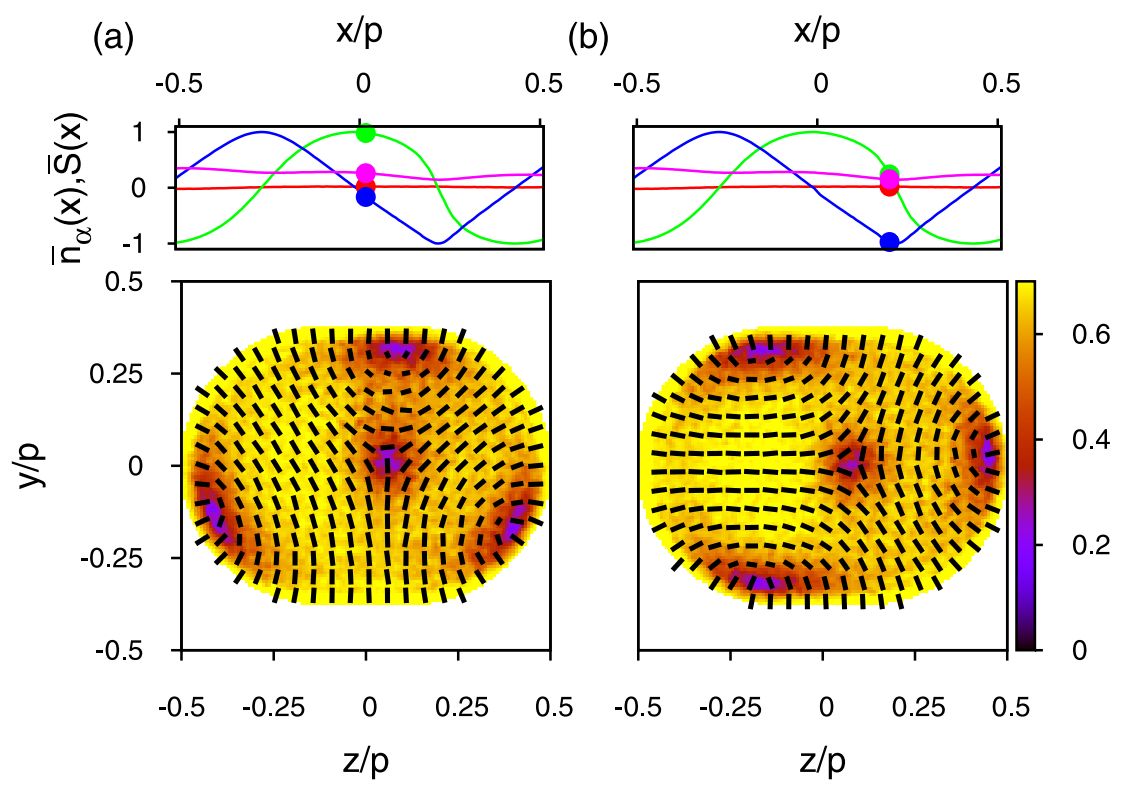

FIG. 14. As Fig. 8, but for $s_{\mathrm{y}} / p=0.75$ and $s_{\mathrm{z}} / p=1.00$. 
(a)

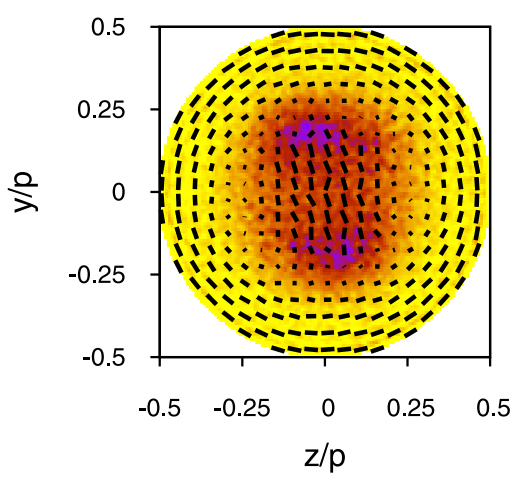

(a)

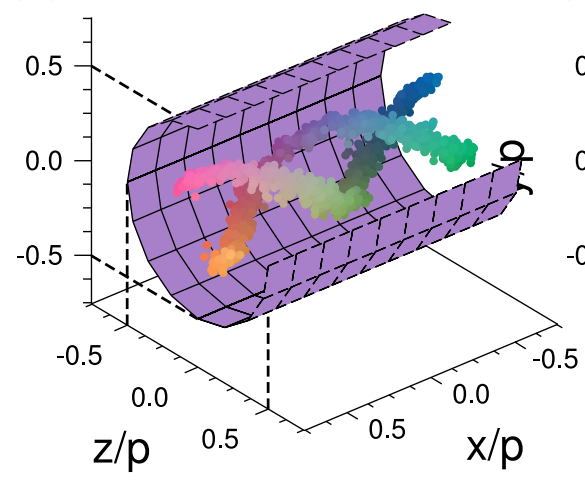

(b)

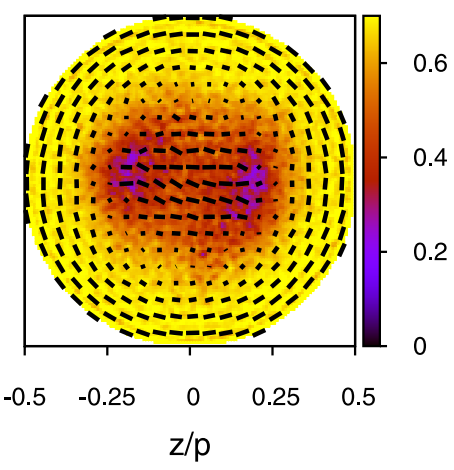

(b)

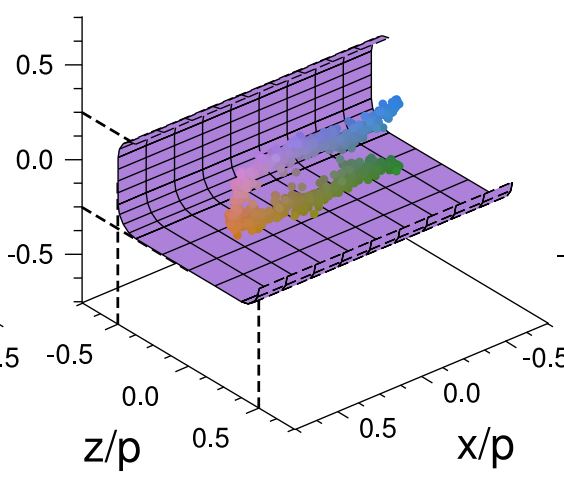

FIG. 15. As Fig. 11, but for locally planar anchoring of mesogens at the walls of the mesochannel.

FIG. 16. As Fig. 9, but for local planar anchoring of mesogens at the wall of a mesochannel. (a) $s_{\mathrm{y}} / p=s_{\mathrm{z}} / p=1.00, R / s_{\mathrm{y}}=0.50$, (b) $s_{\mathrm{y}} / p=0.50, s_{\mathrm{z}} / p=1.00$, $R / s_{\mathrm{y}}=0.25$, (c) $s_{\mathrm{y}} / p=1.00, s_{\mathrm{z}} / p=1.25, R / s_{\mathrm{y}}=0.50$.

If the cylindrical mesochannel is twice as wide, two double-twist helices can be accommodated. In this case, mesogens in regions shared by both double-twist helices are orientationally frustrated such that between the helices, a relatively large region of low $S\left(\boldsymbol{r}^{\prime}\right)$ arises as the plot in Fig. 15 reveals. The two defect regions visible in Fig. 15 form two independent helices along the line of vision as one can see from the plot of disclination lines in three dimensions shown in Fig. 16(a). The helical disclination lines are wrapped around the $x$-axis rotating in a counterclockwise fashion. Exactly the same structure has been reported earlier by Fukuda and Žumer but for a blue phase I in a slit-pore with homeotropic anchoring of the mesogens. ${ }^{31}$

Next, we consider a situation in which the mesochannel is deformed such that it is stretched in the $z$-direction by a factor of two relative to the cross section along the $y$-axis. Under these conditions, the plot in Fig. 17 reveals that two double-twist helices can be accommodated whose centers are approximately located at $y / p=0.00$ and $z / p= \pm 0.25$. Because of the dimensions of the mesochannel chosen here and because of the locally planar anchoring at its walls, no defects arise except in those regions where the doubletwist helices begin to merge along the line $z / p=0.00$. Therefore, one anticipates two separate, linear disclination lines parallel to $\widehat{\boldsymbol{e}}_{\mathrm{x}}$. The plot in Fig. 16(b) confirms this expectation.

The last case considered in this section is that of a mesochannel with an inner surface that is always curved (see Fig. 18). This mesochannel is wide enough so that a trigonal arrangement of three double-twist helices can be

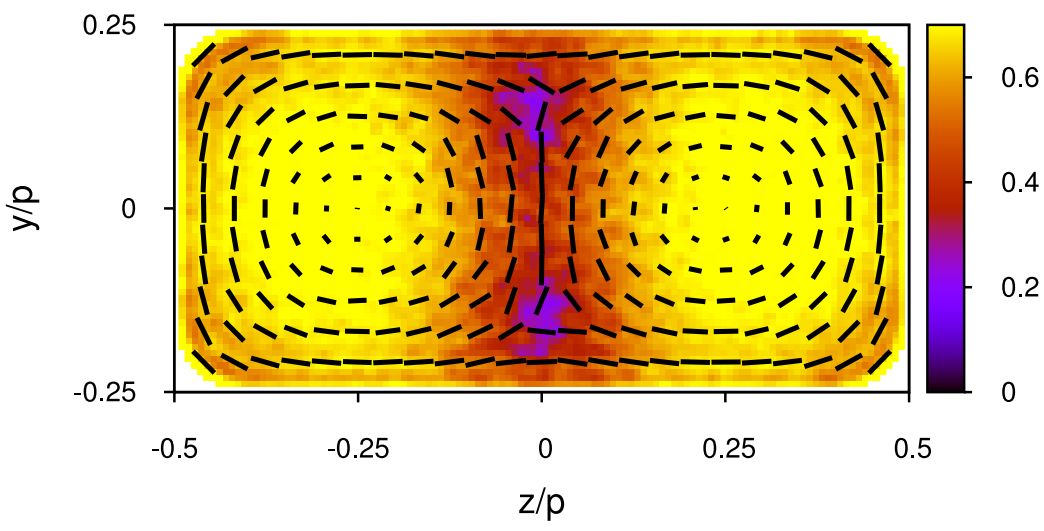

FIG. 17. As Fig. 15, but for $s_{\mathrm{y}} / p=0.50, s_{\mathrm{z}} / p=1.00$, and $R / s_{\mathrm{y}}=0.25$. 
(a)

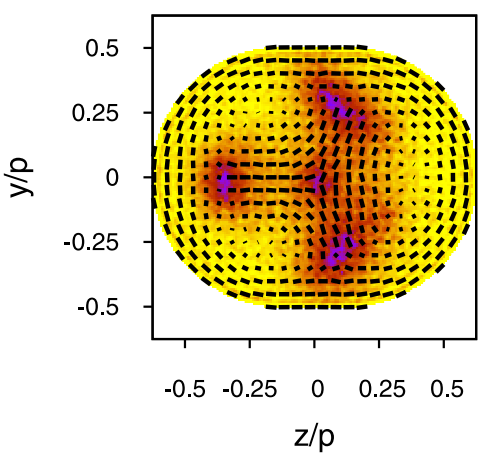

(b)

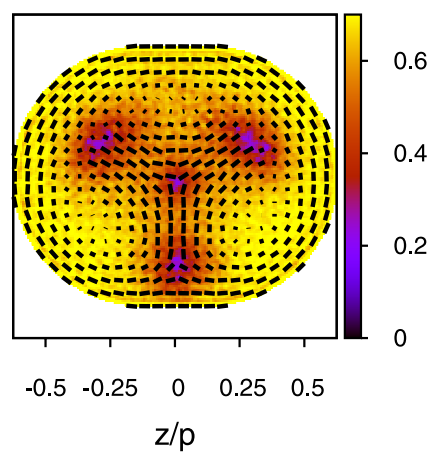

FIG. 18. As Fig. 15, but for $s_{\mathrm{y}} / p=1.00, s_{\mathrm{z}} / p=1.25$, and $R / s_{\mathrm{y}}=0.50$. accommodated. Mesogens are orientationally frustrated in small regions where the circumferences of any two of the three double-twist helices meet. Consequently, three defect regions exist that are rotating in a counterclockwise fashion together with the three double-twist helices. Moreover, we note that this defect topology is structurally equivalent to the one already illustrated in Fig. 14.

In addition, a fourth region of low $S\left(\boldsymbol{r}^{\prime}\right)$ is visible in Fig. 18 surrounded by the trigonal arrangement of the outer ones. In this region, the circumferences of all three double-twist helices gather. This fourth region stays put along $\widehat{\boldsymbol{e}}_{\mathrm{x}}$ as one can see from the plot of $\ell$ in Fig. 16(c). The structure of $\ell$ illustrated by plots in Figs. 16(c) and 18 has already been reported earlier (see Fig. 10(a) of Ref. 28) but for a true cylinder rather than a slightly deformed one considered here.

\section{Directional anchoring}

After having discussed the case of planar anchoring in Sec. IV C 2, we conclude the presentation of results by considering very briefly the case of directional anchoring where the anchoring function is now given by Eq. (3.13). Hence, mesogens located in the immediate vicinity of the walls of the mesochannel tend to align their longer axes with the line of vision $\widehat{\boldsymbol{e}}_{\mathrm{x}}$; any other orientation would receive an energy penalty.

The disclination lines depicted in Fig. 19(a) for a cylindrical mesochannel turn out to be very similar to the ones observed earlier for degenerate planar anchoring [see Fig. 16(c)] and for homeotropic anchoring [see Fig. 12(c)].
However, in these cases, three helices of disclination lines winding around a straight portion of the disclination line along the $x$-axis are only observed for mesochannels for which the cross sections along the $y$ - and $z$-directions are different. In the present case, this configuration is observed for cylindrical mesochannels.

However, two more interesting cases are illustrated by the plots in Figs. 19(b) and 19(c). In both cases, we find ringlike disclination-line loops that are intertwined in ways that depend on the width (i.e., $s_{\mathrm{y}}$ ) of the mesochannels. From the plot in Fig. 19(b), these rings do not intersect at any point in space. This is different for the situation depicted in Fig. 19(c) where we consider a mesochannel that has a larger cross section in the $y$-direction compared with the mesochannel considered in Fig. 19(b). Widening the cross section still causes ringlike defect loops to be stable. However, now these individual loops connect at certain tetrahedrally coordinated points at which pairs of disclination loops merge.

Because it is a formidable task to visualize disclination rings, we amend the plot in Fig. 19(b) by the director field and the associated local nematic order parameter both of which are presented in Fig. 20. One clearly sees the formation of doubletwist helices that have their centers in regions where the dashes representing $\widehat{\boldsymbol{n}}(x, z)$ vanish. The double-twist helices are evolving in the $y$-direction until they reach the walls of the mesochannel. Positions at which the disclination rings are intersecting the $x-z$ plane at $y=0$ manifest themselves as two-dimensional, periodically arranged regions of low nematic order in Fig. 20. Hence, disclination rings are bent with respect to the $x-z$ plane at $y=0$. (a)

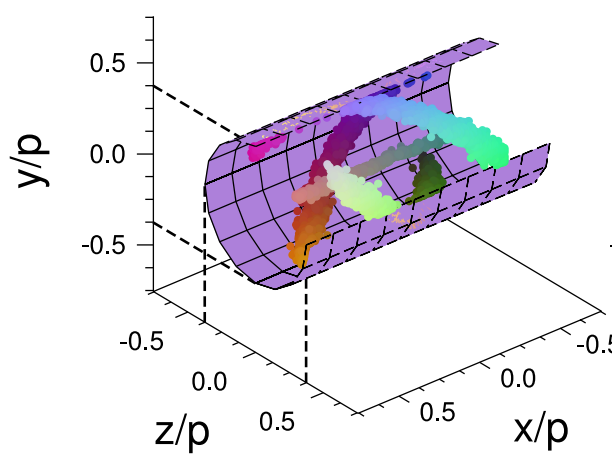

(b)

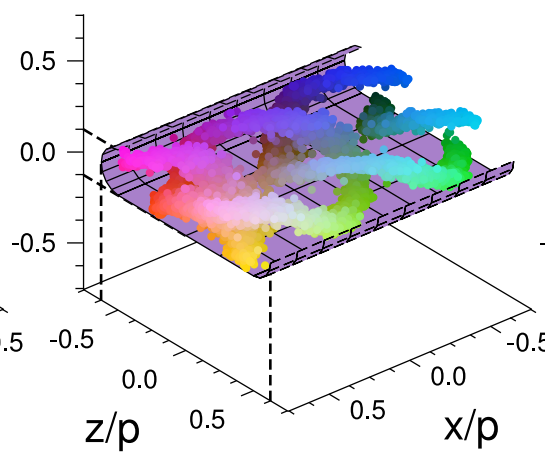

(c)

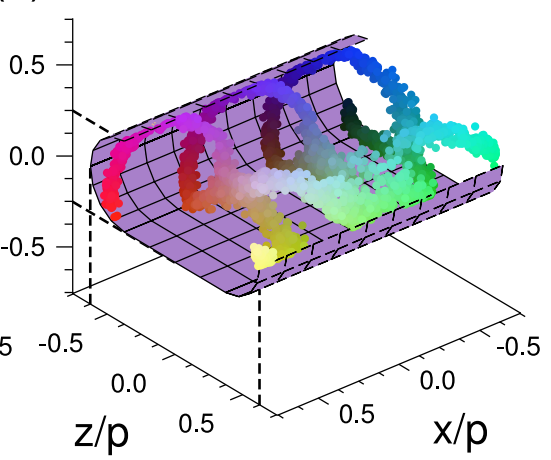

FIG. 19. As Fig. 9, but for local directional anchoring of mesogens at the wall of a mesochannel for a wall curvature of $R / s_{\mathrm{y}}=0.50$. (a) $s_{\mathrm{y}} / p=s_{\mathrm{z}} / p=0.75$, (b) $s_{\mathrm{y}} / p=0.25, s_{\mathrm{z}} / p=1.25$, (c) $s_{\mathrm{y}} / p=0.50, s_{\mathrm{z}} / p=1.25$. 


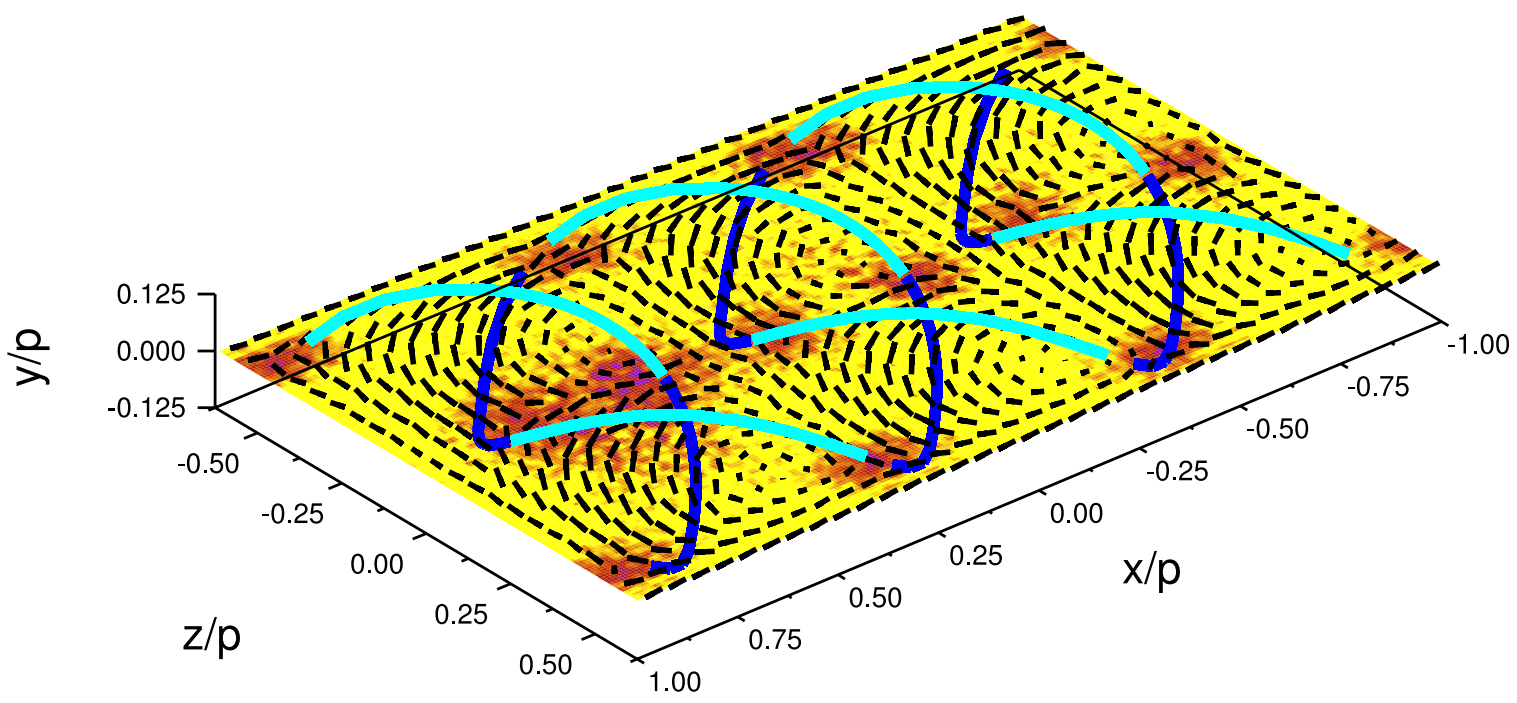

FIG. 20. As Fig. 19(b), but for the local director field (dashes) and the local nematic order parameter. In addition, disclination rings are shown that are colored in cyan if they lie above the $x-z$ plane at $y=0$ and are colored in blue otherwise.

\section{SUMMARY AND CONCLUSIONS}

In this work, we employ MC simulations in the grand canonical and canonical ensembles to investigate a chiral liquid crystal confined to mesochannels. These mesochannels constitute confinement to two dimensions ( $y$ - and $z$ directions) but are quasi-infinite in the $x$-direction on account of periodic boundary conditions. The mesochannels have variable cross sections in the $y-z$ plane which allows us to consider various geometries. The confined mesogens are anchored at the inner surfaces of the mesochannels in three different ways. Specifically, we consider local homeotropic anchoring as well as planar and directional anchorings.

Throughout this work, we selected a thermodynamic state and a strength of the chiral coupling constant in the mesogenmesogen interaction potential such that in the bulk, the liquid crystal is stable in blue phase II. The structure of this phase is characterized by a three-dimensional simple-cubic lattice of double-twist helices that render the liquid crystal highly ordered locally but isotropic globally because the arrangement of helices is the same regardless of the line of vision along the $x$-, $y$-, or $z$-axis.

If this liquid crystal is confined to a mesochannel, the dimensions of its cross section compete with $p$ such that novel defects and disclination-line geometries arise that have no counterpart in the bulk. For cylindrical mesochannels (i.e., for $s_{\mathrm{y}} / s_{\mathrm{z}}=1$ ) with homeotropic wall anchoring and $s_{\mathrm{y}} / p=0.50$, a quasi-cholesteric phase is observed. Here, the local director field indicates that mesogens lie in the $y-z$ plane everywhere along $\widehat{\boldsymbol{e}}_{\mathrm{x}}$. These mesogens are pointing more or less in the same direction such that for each of the $y-z$ planes along the line of vision, the director field is constant.

However, on account of the local homeotropic anchoring at the walls of the mesochannels, small regions exist in which the mesogens are orientationally frustrated. Both the director field and the defects near the walls rotate in the $y-z$ plane along $\widehat{\boldsymbol{e}}_{\mathrm{x}}$. In other words, the confined mesogens form a quasi-cholesteric phase in which the defects form two isolated helices rotating around the line of vision.
If the size of cross sections is maintained but their geometry is changed from cylindrical to rectangular, one observes a similar quasi-cholesteric phase. However, here the defect lines align themselves with the walls. This alignment changes periodically between the two opposite walls of the channel in the $y$ - and $z$-directions, respectively. Thus, unlike for the cylindrical mesochannel, we observe a ladder-like structure of disclination lines in this case. These ladders are periodically connected by other parts of the disclination lines.

In both cases, the double-twist helical structure characteristic of the bulk liquid crystal is suppressed because of the dominant impact of wall anchoring. If the cross section of the cylindrical mesochannel is widened (i.e., for $\left.s_{\mathrm{y}} / p=1.00\right)$, a single double-twist helix forms with its center coinciding with the center of the mesochannel regardless of whether the mesochannel is cylindrical or rectangular in shape. For the cylindrical mesochannel, mesogens appear to be orientationally frustrated in three regions on the circumference of the double-twist helix. Here, the planar orientation of the mesogens in the $y-z$ plane is in conflict with the homeotropic wall anchoring. The three regions form a trigonal structure that rotates in the $y-z$ plane along $\widehat{\boldsymbol{e}}_{\mathrm{x}}$ thereby forming three independent disclination line helices. The arrangement of all six disclination line helices (three inner and three outer ones) forms a perfectly symmetric structure along the cylindrical mesochannel's line of symmetry.

For the same size of the cross section but a rectangular rather than cylindrical geometry, one observes the same ladder-like structure described above. However, because the mesochannel is wider now, a single double-twist helix resides at the center of the mesochannel. Because the inplane orientation of the mesogens at the circumference of the double-twist helix clashes with the locally homeotropic wall anchoring, two regions exist in which the mesogens are orientationally frustrated such that along $\widehat{\boldsymbol{e}}_{\mathrm{x}}$ two helical disclination lines can form.

If the mesochannel is deformed such that the ratio of $s_{\mathrm{y}} / p$ is no longer an integer multiple of $p / 2$ but sufficiently wide, a trigonal arrangement of three partial double-twist helices can 
be accommodated. These double-twist helices are incomplete because of their close proximity to the walls. Nevertheless, they are rotating in the $y-z$ plane as one moves along $\widehat{\boldsymbol{e}}_{\mathrm{x}}$. Near the center of the mesochannel, at which the circumferences of all three double-twist helices meet, a small defect region exists in which the mesogens are orientationally frustrated because here they cannot attain an orientation compatible with that of all three double-twist helices at the same time. This inner region is part of a straight portion of $\ell$ along $\widehat{\boldsymbol{e}}_{\mathrm{x}}$.

Combinations of helical disclination lines and straight ones are also observed for planar wall anchoring. Perhaps most remarkable in this case is a disclination line structure that looks topologically similar to the one described before. However, here the rotating outer disclination lines do not form at the center of each of the three double-twist helices but in regions in which the circumferences of a pair of double-twist helices meet.

Last but not least, if we consider the case of local directional anchoring and mesochannels with a rather substantial shape anisotropy, we are able to stabilize disclination lines that have a ring topology. Ringlike disclination lines have been observed also by Fukuda and Žumer within the framework of Landau-de Gennes theory. ${ }^{32}$ The difference between their disclination rings and ours is that in our case, the rings are intertwined whereas they remain independent and symmetrically arranged entities in the work of Fukuda and Žumer. Moreover, if the aspect ratio of the mesochannel's is a bit larger, the rings persist but now they are connected at isolated, tetrahedrally coordinated "points."

It is particularly gratifying that the present very simple model of a liquid crystal is apparently capable not only to reproduce structural features of bulk chiral phases ${ }^{33,34}$ in a qualitatively correct fashion but also some of the defect topologies reported earlier theoretically, ${ }^{28}$ where a non-molecular approach has been used, as well as one topology that has been seen experimentally quite some time ago. ${ }^{25}$ However, it needs to be stressed again that simply upscaling the topologies observed here to, say, a $\mu \mathrm{m}$ length scale is not possible quantitatively but only qualitatively as already stated in Sec. I.

Thus, we have demonstrated here that if a chiral liquid crystal is confined to containers whose dimensions compete with the pitch of helical structures the liquid crystal would attain in the bulk, a great variety of different disclination line topologies may be observed. Thus, one may envision a great complexity of spatial arrangements of nanoparticles that are brought into contact with these disclination line topologies ${ }^{13}$ which may, in turn, give rise to new nanocolloidal materials with specific and novel functionalities.

\section{ACKNOWLEDGMENTS}

We are grateful to Deutsche Forschungsgemeinschaft for funding under the auspices of the International Graduate Research Training Group 1524 "Self-assembled soft matter nanostructures at interfaces."

${ }^{1}$ J. Planer, Liebigs Ann. Chem. 118, 25 (1861).

${ }^{2}$ P. G. de Gennes and J. Prost, The Physics of Liquid Crystals, 2nd ed. (Clarendon, Oxford, 1995).
${ }^{3}$ W. Cao, A. Muñoz, P. Palffy-Muhoray, and B. Taheri, Nat. Mater. 1, 111 (2002).

${ }^{4}$ S. Yokoyama, S. Machiko, H. Kikuchi, K. Uchida, and T. Nagamura, Adv. Mater. 18, 48 (2006).

${ }^{5}$ H. J. Coles and M. N. Pivnenko, Nature 436, 997 (2005).

${ }^{6}$ H. Kikuchi, M. Yokota, Y. Hisakado, H. Yang, and T. Kajiyama, Nat. Mater. 1, 64 (2002)

${ }^{7}$ F. Castles, F. V. Day, S. M. Morris, D.-H. Ko, D. J. Gardiner, M. M. Qasim, S. Nosheen, P. J. W. Hands, S. S. Choi, R. H. Friend, and H. J. Coles, Nat. Mater. 11, 599 (2012).

${ }^{8}$ Z. Zheng, D. Sheng, and P. Huang, New J. Phys. 12, 113018 (2010).

${ }^{9}$ W. He, G. Pan, Z. Yang, D. Zhao, G. Niu, W. Huang, X. Yuan, J. Guo, H. Cao, and H. Yang, Adv. Mater. 21, 2050 (2009).

${ }^{10}$ A. Yoshizawa, Y. Kogawa, K. Kobayashi, Y. Takanishi, and J. Yamamoto, J. Mater. Chem. 19, 5759 (2009).

${ }^{11}$ T. N. Oo, T. Mizunuma, Y. Nagano, H. Ma, Y. Ogawa, Y. Haseba, H. Higuchi, Y. Okumura, and H. Kikuchi, Opt. Mater. Express 1, 1502 (2011).

${ }^{12}$ H. Yoshida, Y. Tanaka, K. Kawamoto, H. Kubo, T. Tsuda, A. Fujii, S. Kuwabata, H. Kikuchi, and M. Ozaki, Appl. Phys. Express 2, 121501 (2009).

${ }^{13}$ M. Ravnik, G. P. Alexander, J. M. Yeomans, and S. Žumer, Proc. Natl. Acad. Sci. U. S. A. 108, 5188 (2011).

${ }^{14}$ K. Stratford, O. Henrich, J. S. Lintuvuori, M. E. Cates, and D. Marenduzzo, Nat. Commun. 5, 1 (2014).

${ }^{15}$ J.-I. Fukuda and S. Žumer, Phys. Rev. E 87, 042506 (2013).

${ }^{16}$ O. Trushkevychet, P. Ackerman, W. A. Crossland, and I. I. Smalyukh, Appl. Phys. Lett. 97, 201906 (2010).

${ }^{17}$ P. J. Ackerman, Z. Qi, and I. I. Smalyukh, Phys. Rev. E 86, 021703 (2012).

${ }^{18}$ J. S. Evans, P. J. Ackerman, D. J. Broer, J. van de Lagemaat, and I. I. Smalyukh, Phys. Rev. E 87, 032503 (2013).

${ }^{19}$ P. J. Ackerman, R. P. Trivedi, B. Senyuk, J. van de Lagemaat, and I. I. Smalyukh, Phys. Rev. E 90, 012505 (2014).

${ }^{20}$ M. B. Pandey, T. Porenta, J. Brewer, A. Burkart, S. Čopar, S. Žumer, and I. I. Smalyukh, Phys. Rev. E 89, 060502(R) (2014).

${ }^{21}$ F. Hoffmann, M. Cornelius, J. Morell, and M. Fröba, Angew. Chem., Int. Ed. 45, 3216 (2006).

${ }^{22}$ Y. Wan and D. Zhao, Chem. Rev. 107, 2821 (2007).

${ }^{23}$ M. Steinhart, C. Liang, G. W. Lynn, U. Gösele, and S. Dai, Chem. Mater. 19, 2383 (2007).

${ }^{24}$ D. I. Fried, F. J. Brieler, and M. Fröba, ChemCatChem 5, 862 (2013).

${ }^{25}$ P. E. Cladis, A. E. White, and W. F. Brinkman, J. Phys. (Paris) 40, 325 (1979).

${ }^{26}$ F. Lequeux and M. Kléman, J. Phys. (Paris) 49, 845 (1988).

${ }^{27}$ R. J. Ondris-Crawford, M. Ambrožič, and S. Žumer, Phys. Rev. E 50, 4773 (1994)

${ }^{28}$ J. Bezić and S. Žumer, Liq. Cryst. 14, 1695 (1993).

${ }^{29}$ M. Ambrožič and S. Žumer, Phys. Rev. E 54, 5187 (1996).

${ }^{30}$ H. S. Kitzerow, B. Liu, F. Xu, and P. P. Crooker, Phys. Rev. E 54, 568 (1996).

${ }^{31}$ J.-I. Fukuda and S. Žumer, Phys. Rev. Lett. 104, 017801 (2010).

${ }^{32}$ J.-I. Fukuda and S. Žumer, Phys. Rev. Lett. 106, 097801 (2011).

${ }^{33}$ M. Melle, M. Theile, C. K. Hall, and M. Schoen, Int. J. Mol. Sci. 14, 17584 (2013).

${ }^{34}$ M. Melle, S. Schlotthauer, C. K. Hall, E. Díaz-Herrera, and M. Schoen, Soft Matter 10, 5489 (2014).

${ }^{35}$ J. S. Lintuvuori, K. Stratford, M. E. Cates, and D. Marenduzzo, Phys. Rev. Lett. 105, 178302 (2010).

${ }^{36}$ V. S. R. Jampani, M. Škarabot, M. Ravnik, S. Čopar, S. Žumer, and I. Muševič, Phys. Rev. E 84, 031703 (2011).

${ }^{37}$ R. Eppenga and D. Frenkel, Mol. Phys. 52, 1303 (1984).

${ }^{38}$ I. Pardowitz and S. Hess, Physica A 100, 540 (1980).

${ }^{39}$ W. H. Press, S. A. Teukolsky, W. T. Vetterling, and B. P. Flannery, Numerical Recipes: The Art of Scientific Computing, 3rd ed. (Cambridge University Press, 2007), Chap. 11.1.

${ }^{40}$ A. Richter and T. Gruhn, J. Chem. Phys. 125, 064908 (2006).

${ }^{41}$ M. Greschek, M. Melle, and M. Schoen, Soft Matter 6, 1898 (2010).

${ }^{42}$ M. Greschek and M. Schoen, Phys. Rev. E 83, 011704 (2011).

${ }^{43}$ R. Memmer, H.-G. Kuball, and A. Schönhofer, Liq. Cryst. 15, 345 (1993).

${ }^{44}$ H. Steuer, S. Hess, and M. Schoen, Physica A 328, 322 (2003).

${ }^{45}$ W. Maier and A. Saupe, Z. Naturforsch., A: Phys. Sci. 15, 287 (1960).

${ }^{46}$ G. W. Gray and D. G. McDonnell, Mol. Cryst. Liq. Cryst. 34, 211 (1977).

${ }^{47}$ R. S. Pindak, C.-C. Huang, and J. T. Ho, Phys. Rev. Lett. 32, 43 (1974)

${ }^{48}$ S. Giura and M. Schoen, Phys. Rev. E 90, 022507 (2014). 
${ }^{49}$ C. G. Gray and K. E. Gubbins, Theory of Molecular Fluids, Volume 1 (Clarendon, Oxford, 1984).

${ }^{50}$ R. Berardi, H.-G. Kuball, R. Memmer, and C. Zannoni, J. Chem. Soc., Faraday Trans. 94, 1229 (1998).

${ }^{51}$ W. Maier and A. Saupe, Z. Naturforsch., A: Phys. Sci. 13, 564 (1958).

${ }^{52}$ W. Maier and A. Saupe, Z. Naturforsch., A: Phys. Sci. 14, 882 (1959).

${ }^{53}$ R. Memmer, H.-G. Kuball, and A. Schönhofer, Mol. Phys. 89, 1633 (1996).

${ }^{54}$ S. Hess and B. Su, Z. Naturforsch., A: Phys. Sci. 54, 559 (1999)

${ }^{55}$ J. A. Castellano, Mol. Cryst. Liq. Cryst. 94, 33 (1983).
${ }^{56}$ A. A. Sonin, The Surface Physics of Liquid Crystals (Gordon and Breach, Amsterdam, 1995).

${ }^{57}$ B. Jérôme, Rep. Prog. Phys. 54, 391 (1991).

${ }^{58}$ K. S. Shing and S. T. Chung, J. Phys. Chem. 91, 1674 (1987).

${ }^{59}$ T. Gruhn and M. Schoen, Phys. Rev. E 55, 2861 (1997).

${ }^{60}$ J.-I. Fukuda and S. Žumer, Phys. Rev. E 84, 040701 (2011).

${ }^{61}$ J. E. Adams and W. Haas, Mol. Cryst. Liq. Cryst. 15, 27 (1971).

${ }^{62}$ M. R. Wilson and D. J. Earl, J. Mater. Chem. 11, 2672 (2001).

${ }^{63}$ G. P. Crawford and S. Žumer, Liquid Crystals in Complex Geometries (Taylor and Francis, London, 1996). 